\title{
HEAVY-TRAFFIC LIMITS FOR NEARLY DETERMINISTIC QUEUES
}

\author{
KARL SIGMAN *** AND \\ WARD WHITT, ${ }^{* * *}$ Columbia University
}

\begin{abstract}
We establish heavy-traffic limits for nearly deterministic queues, such as the $\mathrm{G} / \mathrm{D} / n$ many-server queue. Since waiting times before starting service in the $G / D / n$ queue are equivalent to waiting times in an associated $\mathrm{G}_{n} / \mathrm{D} / 1$ model, where the $\mathrm{G}_{n}$ interarrival times are the sum of $n$ consecutive interarrival times in the original model, we focus on the $\mathrm{G}_{n} / \mathrm{D} / 1$ model and the generalization to $\mathrm{G}_{n} / \mathrm{G}_{n} / 1$, where 'cyclic thinning' is applied to both the arrival and service processes. We establish different limits in two cases: (i) when $\left(1-\rho_{n}\right) \sqrt{n} \rightarrow \beta$ as $n \rightarrow \infty$ and (ii) when $\left(1-\rho_{n}\right) n \rightarrow \beta$ as $n \rightarrow \infty$, where $\rho_{n}$ is the traffic intensity in model $n$. The nearly deterministic feature leads to interesting nonstandard scaling.

Keywords: Heavy traffic; nearly deterministic queue; Erlang queue; many-server queue; deterministic service time; Gaussian random walk; cyclic thinning; functional central limit theorem; invariance principle
\end{abstract}

2010 Mathematics Subject Classification: Primary 60K25

Secondary 60F17; 90B22

\section{Introduction}

A primary cause of congestion in a queueing system is stochastic fluctuations in the arrival times and the service times. We say that a queueing system is nearly deterministic if these stochastic fluctuations are low. At customary loads, the congestion in a nearly deterministic queueing system will be negligible. However, if the system is nearly deterministic then it is natural to operate the system at higher loads. In this paper we explore the interplay between low variability and high loads. In particular, we establish heavy-traffic (HT) limits for some nearly deterministic queueing models.

A classic example of a nearly deterministic queueing model is the $\mathrm{GI} / \mathrm{D} / n$ multiserver queue when $n$ is large. The GI/D/n model has $n$ homogeneous servers working in parallel, an unlimited waiting room, the first-come-first-served (FCFS) service discipline, identical deterministic service times, and a renewal arrival process with a general interarrival-time distribution. It is well known that waiting times (before starting service) in this model can be identified with waiting times in the corresponding $\mathrm{GI}_{n} / \mathrm{D} / 1$ model, where the $\mathrm{GI}_{n}$ means that the arrival process is the renewal process whose interarrival times are distributed as the sum of $n$ interarrival times in the original renewal arrival process; see, e.g. Theorem 4.6.1 of [13]. That occurs because, without loss of generality, the customers can be assigned to the servers in a round robin or cyclic order. For large $n$, these $\mathrm{GI}_{n} / \mathrm{D} / 1$ models become nearly deterministic, approaching the

Received 11 May 2010; revision received 17 April 2011.

* Postal address: Department of Industrial Engineering and Operations Research, Columbia University, New York, NY 10027-6699, USA.

** Email address: ks20@ columbia.edu

*** Email address: ww2040@columbia.edu 
$\mathrm{D} / \mathrm{D} / 1$ queue. Of course, the service times are completely deterministic from the outset, but also the $\mathrm{GI}_{n}$ interarrival times become nearly deterministic as $n$ increases by virtue of the law of large numbers. For example, if the original GI interarrival times have squared coefficient of variation (variance divided by the square of the mean) $c_{a}^{2}$, then the $\mathrm{GI}_{n}$ interarrival times have squared coefficient of variation $c_{a}^{2} / n$, which converges to 0 as $n \rightarrow \infty$.

In applications, a Poisson arrival process is often a realistic assumption. The reduction of $\mathrm{M} / \mathrm{D} / n$ to $\mathrm{E}_{n} / \mathrm{D} / 1$ for the waiting times is often mentioned in textbooks. Otherwise, the renewal process assumption is not so realistic. Thus, it is important that both the reduction of the GI/D/n model to the $\mathrm{GI}_{n} / \mathrm{D} / 1$ model and our HT limits hold for more general ' $\mathrm{G}$ ' arrival processes. There are no algorithms available to compute the steady-state waiting time distribution or even only its mean in the new $\mathrm{G}_{n} / \mathrm{D} / 1$ model. Thus, the simple approximations stemming from the HT limits we establish here can be very useful. Consistent with the large body of HT literature, we only assume that the general $\mathrm{G}$ arrival counting process or, equivalently, the associated partial sums of consecutive interarrival times, satisfies a functional central limit theorem (FCLT); see Section 4.4 of [14] for examples with dependence that are covered.

Motivated by the example above, we will consider the waiting time process in single-server queues with the $\mathrm{G}_{n} / \mathrm{G}_{n} / 1$ structure, where cyclic thinning is applied to both the interarrival times and the service times. Our results cover the two models $\mathrm{D} / \mathrm{G}_{n} / 1$ and $\mathrm{G}_{n} / \mathrm{D} / 1$ as special cases, because $G_{n}$ coincides with the original $G$ when the $G$ is $D$; i.e. $D_{n}=D$. That is so because the deterministic renewal process (D) is the unique fixed point among renewal processes of the operation mapping GI into $\mathrm{GI}_{n}$, when we rescale to fix the mean. (Uniqueness follows immediately from the squared coefficient of variations.) When working with partial sums of interarrival times or service times, we let the new sequence of $\mathrm{G}_{n}$ partial sums $\left\{S_{n, k}: k \geq 1\right\}$ be defined in terms of the sequence of original G partial sums $\left\{S_{1, k}: k \geq 1\right\}$ by letting $S_{n, k} \equiv$ $S_{1, k n} / n, k \geq 1$; i.e. we scale the index $k$ in the original partial sums $S_{1, k}$ by $n$ because we add $n$ consecutive times, but we also divide by $n$ in order to keep the mean fixed in the identically distributed case. It is easy to see that $\mathrm{D}_{n}=\mathrm{D}$ with this construction.

If the traffic intensity $\rho_{n}$ in the $\mathrm{G}_{n} / \mathrm{G}_{n} / 1$ model (assumed well defined) is held fixed at a stable value or, more generally, satisfies $\rho_{n} \rightarrow \rho<1$ as $n \rightarrow \infty$, then the $\mathrm{G}_{n} / \mathrm{G}_{n} / 1$ model approaches the purely deterministic D/D/1 model, and the stationary waiting time becomes asymptotically negligible. However, we let $\rho_{n} \uparrow 1$ as $n \rightarrow \infty$. We thus obtain an interesting double limit, in which the models approach $\mathrm{D} / \mathrm{D} / 1$, while the traffic intensity increases. On the one hand, congestion should decrease, because the models are becoming less variable, approaching D/D/1. On the other hand, the congestion should increase because we let $\rho_{n} \uparrow 1$. We let $\rho_{n}$ approach 1 at an appropriate rate so that we get revealing nondegenerate limits.

For the multiserver $\mathrm{G} / \mathrm{D} / n$ model mentioned at the outset, the double limit coincides with the familiar many-server HT limit, in which we let the traffic intensities $\rho_{n}$ approach 1 as the number of servers, $n$, increases; see, e.g. [6] and [10]. We consider the so-called Halfin-Whitt or qualityand-efficiency-driven (QED) regime, in which $\left(1-\rho_{n}\right) \sqrt{n} \rightarrow \beta, 0<\beta<\infty$. However, we also consider the case in which $\left(1-\rho_{n}\right) n \rightarrow \beta, 0<\beta<\infty$. In that case, we obtain a nondegenerate limit for the un-normalized waiting times.

The asymptotically deterministic feature is critical for these new limits. For example, the HT limits for the $\mathrm{G}_{n} / \mathrm{GI} / 1$ model as $n \rightarrow \infty$ with fixed service time distribution are significantly different (i) when the GI service time distribution is D and (ii) when the service time distribution is not $\mathrm{D}$ (and we do not perform the cyclic thinning on the service times, replacing GI by $\mathrm{GI}_{n}$ ). When the service time distribution is not deterministic, the $\mathrm{G}_{n} / \mathrm{GI} / 1$ model is not asymptotically deterministic as $n \rightarrow \infty$. As a consequence, the HT limit agrees with the conventional limit 

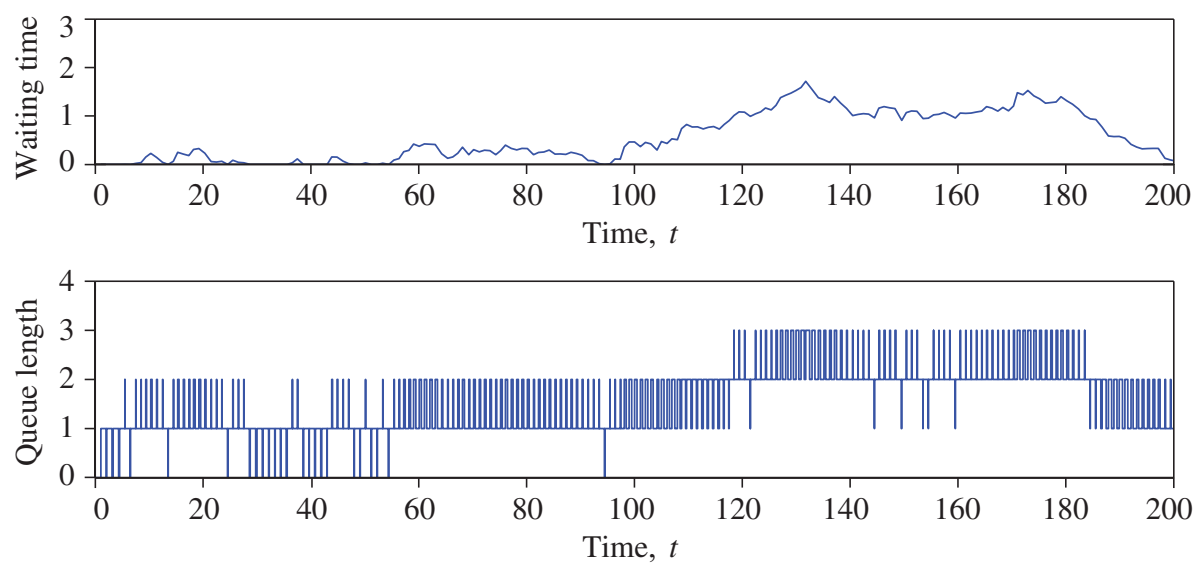

FIGURE 1: Simulation plots of the waiting times at arrival epochs and queue lengths at arbitrary times in the $\mathrm{E}_{100} / \mathrm{D} / 1$ model with $\rho=0.99$, starting empty, for a time interval of length 200 ending at $t=50000$.

for the corresponding D/GI/1 model, with the usual scaling, obtained by simply replacing the interarrival time distribution in the $\mathrm{G}_{n}$ process by deterministic interarrival times with the same mean. In contrast, that is not the case with the nearly deterministic $\mathrm{G}_{n} / \mathrm{D} / 1$ model.

In many ways, the HT behavior of the $\mathrm{G}_{n} / \mathrm{G}_{n} / 1$ models as $n \rightarrow \infty$ is different from the conventional HT behavior of the GI/GI/1 model, discussed in Chapters 5 and 9 of [14]. Unlike the conventional HT theory for the GI/GI/1 model, for the $\mathrm{G}_{n} / \mathrm{G}_{n} / 1$ models there need not be any spatial scaling. In the conventional HT limit, the queue length and waiting time processes have the same asymptotic behavior; both processes behave like reflected Brownian motion, after the same scaling. In contrast, for the $\mathrm{G}_{n} / \mathrm{G}_{n} / 1$ models, the waiting time and queue length processes look very different.

To illustrate these differences, and to provide motivation for the results to follow, we now plot sample paths of the waiting times (before starting service) of successive arrivals and the continuous-time queue length process from one simulation run of the $\mathrm{E}_{100} / \mathrm{D} / 1$ queue with traffic intensity $\rho=0.99$ and unit service times. Figure 1 shows the waiting times at arrival epochs and the continuous-time queue length process, starting empty, in the final subinterval of length 200 ending at $t=5 \times 10^{4}$ from a single run over the time interval [0, $\left.5 \times 10^{4}\right]$.

First, all values of both processes over the full time interval of length 50000 fall in the interval $[0,5]$ without any spatial scaling. The waiting times are comparable to the unit service times, e.g. the average waiting time is about 0.5 . Second, the waiting time plots look continuous, like a plot of reflected Brownian motion, which we will show is indeed its HT limit. In contrast, the queue length process is integer valued, making frequent jumps of size 1. Evidently, its limiting behavior is more complicated. We will explain these plots in the rest of this paper.

\section{Related literature and organization}

\subsection{Three motivating precedents}

In doing this work, we were motivated by three precedents in the literature: (i) the 1993 paper by Abate et al. [1], (ii) the 1996 paper by Song and Zipkin [12], and (iii) the 2004 paper by Jelenkovic et al. [7]. 
In [1] the authors developed an algorithm to compute the distribution and its cumulants of the steady-state waiting time distribution in general GI/GI/1 queues. In order to demonstrate the power of this algorithm, examples of various models were considered that should be challenging by other methods. In Example 3.1 of [1] the authors considered the high-order Erlang model $\mathrm{E}_{n} / \mathrm{E}_{n} / 1$. The algorithm was applied for $n=10^{k}, k=1, \ldots, 4$. However, it was observed that the waiting time would become negligible unless the traffic intensity $\rho_{n}$ in model $n$ were allowed to increase with $n$. Numerical results in Table 1 of [1] showed that the waiting time distribution converges to a mean- 1 exponential distribution as $n \rightarrow \infty$ when $\left(1-\rho_{n}\right) n=1$ as $n \rightarrow \infty$. Table 1 of [1] showed that the HT approximation is remarkably accurate for the $\mathrm{E}_{n} / \mathrm{E}_{n} / 1$ model with large $n$. At that time, this limiting result was confirmed mathematically using the transform method for establishing HT limits due to Kingman [8]. Here we show that the result generalizes, first, to more general models, second, to transient as well as steady-state waiting times, and, third, to other related processes, such as the queue length.

In [12] the authors studied a basic $(r, q)$ inventory model, in which the demand forms a Poisson process at rate $\lambda$ and the lead times are independent and identically distributed (i.i.d.) as $L$. Every $q$ th demand from the Poisson process triggers an order requiring time $L$ to arrive. Thus, there is an $\mathrm{E}_{q} / \mathrm{GI} / \infty$ queue in the background. They were interested in the joint effect upon performance of $\operatorname{var}(L)$ and the lot size $q$. Because the model is intractable, they used an HT approximation. They first considered the case of deterministic lead times, yielding the $\mathrm{E}_{k} / \mathrm{D} / \infty$ queue. They then applied the standard HT limit for the GI/D/ $\infty$ queue, as in [5]. They then made the observation (on page 1356) that the 'interesting' (e.g. optimal) value for $q$ should be of $O(\sqrt{\lambda})$. That observation suggests considering the joint HT limit in which $\lambda \rightarrow \infty$ and $q \equiv q(\lambda) \rightarrow \infty$ with $q(\lambda)=\sqrt{\lambda}$. However, as in [5], in the G/D/ $\infty$ model the queue length (number of busy servers) at time $t, Q(t)$, can be expressed directly in terms of the arrival counting process $N(t)$ : with unit service times $Q_{q}(t)=N_{q}(t)-N_{q}(t-1)$. Thus, it is natural to ask about limits for the counting process in which $\lambda \rightarrow \infty$ and $q \equiv q(\lambda) \rightarrow \infty$ with $q(\lambda)=\sqrt{\lambda}$. The counting process $\left\{N_{q}(t): t \geq 0\right\}$ itself is interesting, being a deterministic cyclic thinning of a base counting process. We investigate these counting processes here in Section 5. We show that a conventional HT limit does not exist, but unconventional HT limits do exist.

In [7] the authors established an HT limit for the steady-state waiting time in the GI/D/n model. They considered the conventional QED many-server regime in which $\left(1-\rho_{n}\right) \sqrt{n} \rightarrow \beta$, $0<\beta<\infty$. Under that scaling, they obtained a nondegenerate limit for the scaled steadystate waiting time $\sqrt{n} W_{n, \infty}$ (which implies that $W_{n, \infty} \Rightarrow 0$, where ' $\Rightarrow$ ' denotes convergence in distribution). This attracted our attention, because at first it seemed inconsistent with the previous results in [1]. At first, we thought that one must be incorrect, but later we discovered that was not so. We demonstrate that here, by obtaining HT limits in both regimes: $\left(1-\rho_{n}\right) \sqrt{n} \rightarrow \beta$ and $\left(1-\rho_{n}\right) n \rightarrow \beta$.

\subsection{Organization}

In Section 3 we review the framework for the conventional HT limit for the waiting times in the single-server queue. In Section 4 we establish the HT FCLTs for the waiting times in the $\mathrm{G}_{n} / \mathrm{G}_{n} / 1$ model with the two different scalings by exploiting Section 3. In Section 5 we establish limits for associated counting processes. These require further unconventional scaling plus an unconventional space and topology. In Section 6 we obtain HT limits for associated continuous-time processes, such as the workload and queue length. In Section 7 we obtain an $\mathrm{HT}$ FCLT for the $\mathrm{G}_{n} / \mathrm{D} / \infty$ model related to the motivating inventory problem from [12]. 
We have also done related work. In [11] we obtained additional HT limits for the stationary distributions. In [9] we studied the heavily loaded GI/D/n+ GI many-server queue with customer abandonment (the $+\mathrm{GI}$ ). We show that the nearly deterministic nature leads to nearly periodic behavior, which can be understood through a careful study of the limiting deterministic fluid model.

\section{Background for general single-server queues}

The proofs of the HT limits for the $\mathrm{G}_{n} / \mathrm{G}_{n} / 1$ models in Section 4 are shortened by making connections to the conventional HT limit for the G/G/1 model. Hence we review that framework; for more, see Chapters 5 and 9 of [14].

\subsection{The standard double-sequence framework}

Consider a sequence of general single-server queues with unlimited waiting room and the FCFS service discipline, indexed by $n \geq 1$. For $n \geq 1$, let the model be specified by a sequence $\left\{\left(U_{n, k}, V_{n, k-1}\right): k \geq 1\right\}$ of ordered pairs of random variables, with $U_{n, k}$ representing the interarrival time between customers $k-1$ and $k$ and $V_{n, k}$ representing the service time of customer $k$. Let a 0 th customer arrive at time 0 and experience an initial wait $W_{n, 0}$. (That is due to customers initially in the system at time 0 . To describe the waiting times of new customers, we do not need to identify these old customers and their service times.) Let $W_{n, k}$ be the waiting time (before beginning service) of customer $k$ in model $n$, defined recursively by

$$
W_{n, k} \equiv\left[W_{n, k-1}+V_{n, k-1}-U_{n, k}\right]^{+}, \quad k \geq 1,
$$

where $[x]^{+} \equiv \max \{x, 0\}$. As a consequence, the waiting times can be expressed via

$$
W_{n, k}=W_{n, 0}+S_{n, k}-\min _{0 \leq j \leq k}\left\{\left(W_{n, 0}+S_{n, j}\right) \wedge 0\right\}, \quad k \geq 0,
$$

where $a \wedge b \equiv \min \{a, b\}$,

$$
S_{n, k} \equiv X_{n, 1}+\cdots+X_{n, k} \quad \text { for } X_{n, k} \equiv V_{n, k-1}-U_{n, k}, k \geq 1,
$$

with $S_{n, 0} \equiv 0$, so that $S_{n, k}=S_{n, k}^{v}-S_{n, k}^{u}$ with

$$
S_{n, k}^{u} \equiv U_{n, 1}+\cdots+U_{n, k}, \quad S_{n, k}^{v} \equiv V_{n, 0}+\cdots+V_{n, k-1}, \quad k \geq 1,
$$

$S_{n, 0}^{v} \equiv 0$ and $S_{n, 0}^{u} \equiv 0$; see Section 9.2 of [14].

Formula (3.2) is a discrete reflection map, mapping the space $\mathbb{R}^{\infty}$ of sequences $x \equiv\left\{x_{k}: k \geq\right.$ $0\}$ into itself; i.e. $W_{n}=\tilde{\phi}\left(W_{n, 0}+S_{n}\right)$ for $W_{n} \equiv\left\{W_{n, k}: k \geq 0\right\}, S_{n} \equiv\left\{S_{n, k}: k \geq 0\right\}$, and $W_{n, 0}+S_{n} \equiv\left\{W_{n, 0}+S_{n, k}: k \geq 0\right\}$, where $\tilde{\phi}: \mathbb{R}^{\infty} \rightarrow \mathbb{R}^{\infty}$ is defined by

$$
\tilde{\phi}(k) \equiv x_{k}-\min _{0 \leq j \leq k}\left\{x_{j} \wedge 0\right\}, \quad k \geq 0 .
$$

The standard HT limit is for a sequence of random elements in the function space $D \equiv$ $D([0, \infty), \mathbb{R})$ of all right-continuous real-valued functions on the positive half-line with limits from the left everywhere (except at 0 ), endowed with the standard Skorokhod $\left(J_{1}\right)$ topology; see [3] and [14]. The HT limit involves scaling space and time. For a real number $t$, let $\lfloor t\rfloor$ be the floor function, giving the greatest integer less than or equal to $t$. Let random elements 
associated with the sequences above be defined by

$$
\begin{array}{cc}
S_{n}^{u}(t) \equiv \frac{S_{n,\lfloor n t\rfloor}^{u}-\lfloor n t\rfloor}{\sqrt{n}}, \quad S_{n}^{v}(t) \equiv \frac{S_{n,\lfloor n t\rfloor}^{v}-\lfloor n t\rfloor}{\sqrt{n}}, \\
S_{n}(t) \equiv \frac{S_{n,\lfloor n t\rfloor}}{\sqrt{n}}, \quad \text { and } \quad \boldsymbol{W}_{n}(t) \equiv \frac{W_{n,\lfloor n t\rfloor}}{\sqrt{n}} .
\end{array}
$$

Let $D^{k} \equiv D \times \cdots \times D$ be the $k$-fold product space of $D$ with itself; let $C$ and $C^{k}$ be the subsets of continuous functions in $D$ and $D^{k}$, respectively. Let $\phi: D \rightarrow D$ be the one-dimensional reflection map, defined by

$$
\phi(x)(t) \equiv x(t)-\inf _{0 \leq s \leq t}\{x(s) \wedge 0\}, \quad t \geq 0 ;
$$

see Sections 3.5 and 13.5 of [14]. The following result is now well known; see Chapter 9 of [14], especially Theorems 9.3.1 and 9.3.3.

Theorem 3.1. (HT limit for the waiting times in $G / G / 1$ models.) If

$$
\left(\boldsymbol{W}_{n}(0), \boldsymbol{S}_{n}^{u}, \boldsymbol{S}_{n}^{v}\right) \Rightarrow\left(\boldsymbol{W}(0), L^{u}, L^{v}\right) \quad \text { in } \mathbb{R} \times D^{2},
$$

where $\mathrm{P}\left(\left(L^{u}, L^{v}\right) \in C^{2}\right)=1$, then

$$
\left(\boldsymbol{S}_{n}^{u}, \boldsymbol{S}_{n}^{v}, \boldsymbol{S}_{n}, \boldsymbol{W}_{n}\right) \Rightarrow\left(L^{u}, L^{v}, L, \boldsymbol{W}\right) \text { in } D^{4} \text { as } n \rightarrow \infty,
$$

where $L \equiv L^{v}-L^{u}, \boldsymbol{W} \equiv \phi(\boldsymbol{W}(0)+L)$, and the limit is in $C^{4}$ with probability 1 .

Proof. Apply the continuous mapping theorem with the addition and reflection functions, because $\boldsymbol{W}_{n}(0)+\boldsymbol{S}_{n}=\boldsymbol{W}_{n}(0)+\boldsymbol{S}_{n}^{v}-\boldsymbol{S}_{n}^{u}$ and $\boldsymbol{W}_{n}=\phi\left(\boldsymbol{W}_{n}(0)+\boldsymbol{S}_{n}\right)$.

\subsection{Scaling unit-rate processes}

For simplicity, and without practical loss of generality, we can construct the sequence of sequences $\left\{\left\{\left(U_{n, k}, V_{n, k-1}\right): k \geq 1\right\}: n \geq 1\right\}$ specifying the sequence of queueing models starting from a single sequence of ordered pairs of random variables $\left\{\left(U_{k}, V_{k-1}\right): k \geq 1\right\}$. This simplification is important for us, because we want to simultaneously consider different scaling in a common framework.

Paralleling (3.3), let

$$
S_{k}^{u} \equiv U_{1}+\cdots+U_{k} \quad \text { and } \quad S_{k}^{v} \equiv V_{0}+\cdots+V_{k-1}, \quad k \geq 1,
$$

$S_{0}^{v} \equiv 0$ and $S_{0}^{u} \equiv 0$.

We have not yet specified any specific stochastic properties. As a canonical case, we have in mind the special case in which $\left\{U_{k}: k \geq 1\right\}$ and $\left\{V_{k-1}: k \geq 1\right\}$ are independent sequences of i.i.d. random variables with means $\mathrm{E}\left[U_{k}\right]=\mathrm{E}\left[V_{k}\right]=1$. With that case in mind (but not assumed), we define the usual sequence of random elements of $D$ associated with this sequence $\left(\hat{\boldsymbol{S}}^{u}, \hat{\boldsymbol{S}}^{v}\right) \equiv\left\{\left(\hat{\boldsymbol{S}}_{k}^{u}, \hat{\boldsymbol{S}}_{k}^{v}\right): k \geq 0\right\}$ by

$$
\hat{\boldsymbol{S}}_{n}^{u}(t) \equiv \frac{S_{\lfloor n t\rfloor}^{u}-\lfloor n t\rfloor}{\sqrt{n}} \quad \text { and } \quad \hat{\boldsymbol{S}}_{n}^{v}(t) \equiv \frac{S_{\lfloor n t\rfloor}^{v}-\lfloor n t\rfloor}{\sqrt{n}}, \quad t \geq 0 .
$$

In this context, our basic assumption is that the sequence $\left\{\left(\hat{\boldsymbol{S}}_{n}^{u}, \hat{\boldsymbol{S}}_{n}^{v}\right): n \geq 1\right\}$ converges, i.e. the partial sums satisfy a joint FCLT. 
To construct a sequence of $\mathrm{G} / \mathrm{G} / 1$ models in which the arrival rate and, thus, the traffic intensity are $\rho_{n}$ in model $n$, where $\rho_{n} \uparrow 1$ as $n \rightarrow \infty$, we use the given service time sequence for all $n$ and introduce extra scaling in the interarrival times; i.e. we let

$$
V_{n, k} \equiv V_{k} \quad \text { and } \quad U_{n, k} \equiv \frac{U_{k}}{\rho_{n}} \quad \text { for all } n, k \geq 1,
$$

with the understanding that $0<\rho_{n}<1$ and that we intend to let $\rho_{n} \uparrow 1$ as $n \rightarrow \infty$. We have thus defined a sequence of queueing models as in Section 3.1.

We are now ready to establish the HT limit theorem for the waiting times in this context. For that purpose, let $e$ be the identity function in $D$, i.e. $e(t)=t, t \geq 0$, and let ' $=$ ' denote equality in distribution (as a process). We will also describe the standard special case, which involves standard Brownian motion (BM), which has zero drift and unit diffusion coefficient. Then the HT limit $\phi(L)$ for the waiting times becomes reflected Brownian motion (RBM) with negative drift.

Theorem 3.2. (HT limit in the single-sequence framework.) Suppose that

$$
\left(\boldsymbol{W}_{n}(0), \hat{\boldsymbol{S}}_{n}^{u}, \hat{\boldsymbol{S}}_{n}^{v}\right) \Rightarrow\left(\boldsymbol{W}(0), \hat{L}^{u}, \hat{L}^{v}\right) \quad \text { in } \mathbb{R} \times D^{2}
$$

for $\left(\hat{\boldsymbol{S}}_{n}^{u}, \hat{\boldsymbol{S}}_{n}^{v}\right)$ in (3.9), where $\mathrm{P}\left(\left(\hat{L}^{u}, \hat{L}^{v}\right) \in C^{2}\right)=1$. If

$$
\left(1-\rho_{n}\right) \sqrt{n} \rightarrow \beta, \quad 0 \leq \beta<\infty, \text { as } n \rightarrow \infty,
$$

then the conditions and conclusions of Theorem 3.1 hold with

$$
L^{u}=\hat{L}^{u}+\beta e \text { and } L^{v}=\hat{L}^{v} .
$$

If, in addition,

$$
\left(\hat{L}^{u}, \hat{L}^{v}\right)=\left(\sigma_{u} B_{u}, \sigma_{v} B_{v}\right),
$$

where $\boldsymbol{W}(0), B_{u}$, and $B_{v}$ are mutually independent, and $B_{u}$ and $B_{v}$ are standard $B M s$, then $L \equiv L^{v}-L^{u} \stackrel{\mathrm{D}}{=} \sigma B-\beta e, B$ is a standard $B M$ and $\sigma^{2}+\sigma_{u}^{2}+\sigma_{v}^{2}$, so that limit (3.8) holds with $\boldsymbol{W}_{n} \Rightarrow \boldsymbol{W} \equiv \phi(\boldsymbol{W}(0)+L)=\phi(\boldsymbol{W}(0)+\sigma B-\beta e)$, which is $R B M$ with drift $-\beta$ starting at an independent random initial state $\boldsymbol{W}(0)$. Furthermore, if (3.11) also holds with $\boldsymbol{W}(0)$ exponentially distributed with mean $\sigma^{2} / 2 \beta$, then the limit $\boldsymbol{W}$ is a stationary RBM.

Proof. Under condition (3.12),

$$
\frac{1}{\rho_{n}}=\frac{1}{1-\beta / \sqrt{n}+o(1 / \sqrt{n})}=1+\frac{\beta}{\sqrt{n}}+o\left(\frac{1}{\sqrt{n}}\right)
$$

as $n \rightarrow \infty$. Hence,

$$
S_{n}^{u}(t)=\hat{\boldsymbol{S}}_{n}^{u}(t)+\frac{S_{\lfloor n t\rfloor}^{u}}{n}(\beta+o(1))
$$

as $n \rightarrow \infty$. The assumed FCLT implies a corresponding functional weak law of large numbers (FWLLN), so that $\hat{\boldsymbol{S}}_{n}^{u} / \sqrt{n} \Rightarrow 0 e$ as $n \rightarrow \infty$. Hence, the second term on the right-hand side of (3.16) converges to $\beta e$, so that the conditions of Theorem 3.1 are satisfied with (3.13). The RBM converges to an exponential distribution with mean $\sigma^{2} / 2 \beta$ as $t \rightarrow \infty$. 


\section{Two HT limits for $G_{n} / G_{n} / 1$ models}

In the setting of Section 3.1, involving a sequence of $\mathrm{G} / \mathrm{G} / 1$ queueing models indexed by $n$, we can obtain a sequence of nearly deterministic queueing models if we assume that cyclic thinning is performed on both the interarrival times and the service times for the $n$th queueing model, with the cycle length increasing as $n \rightarrow \infty$. With 'cyclic thinning' of a point process of order $n$, we select every $n$th point; i.e. the $k$ th point in the thinned process is point $k n$ of the original process. In this context, we call $n$ the cycle length. In this section we assume that the cycle length in model $n$ is $n$, and refer to the model as the $\mathrm{G}_{n} / \mathrm{G}_{n} / 1$ model. In this way, we map an original 'base' sequence of $\mathrm{G} / \mathrm{G} / 1$ models into a sequence of $\mathrm{G}_{n} / \mathrm{G}_{n} / 1$ models.

In the framework of Section 3.1, we replace the partial sums $S_{n, k}^{u}$ and $S_{n, k}^{v}$ with new partial sums $S_{n, k}^{c, u}$ and $S_{n, k}^{c, v}$ defined by

$$
S_{n, k}^{c, u} \equiv \frac{S_{n, k n}^{u}}{n} \quad \text { and } \quad S_{n, k}^{c, v} \equiv \frac{S_{n, k n}^{v}}{n} \quad \text { for all } n \geq 1 \text { and } k \geq 1 .
$$

Then let the associated interarrival times and service times be defined in terms of the increments by

$$
U_{n, k}^{c} \equiv S_{n, k}^{c, u}-S_{n, k-1}^{c, u} \quad \text { and } \quad V_{n, k-1}^{c} \equiv S_{n, k}^{c, v}-S_{n, k-1}^{c, v} .
$$

From (4.1) and (4.2), we see that each new interarrival time is the sum of $n$ of the original interarrival times in model $n$, but we also divide the sums by $n$ to leave the means unchanged (in the case of identically distributed random variables).

We also must treat the initial conditions. We assume that the initial conditions do not get transformed by cyclic thinning. Hence, we have $S_{n, 0}^{c} \equiv S_{n, 0} \equiv W_{n, 0}$ for each $n$. We also assume that the initial conditions scale differently. However, there would be no difference if the systems start empty.

In this section we will show that HT limits for a base sequence of $\mathrm{G} / \mathrm{G} / 1$ 'base' models translate into corresponding HT limits for the sequence of $\mathrm{G}_{n} / \mathrm{G}_{n} / 1$ models. We will show that this can be done in two different ways, depending upon the scaling. We will get different limiting behavior in the following two cases.

Case (i): $\left(1-\rho_{n}\right) \sqrt{n} \rightarrow \beta$ as $n \rightarrow \infty$, where $0<\beta<\infty$.

Case (ii): $\left(1-\rho_{n}\right) n \rightarrow \beta$ as $n \rightarrow \infty$, where $0<\beta<\infty$.

Case (i) is the traditional scaling used in Section 3. However, because of the nearly deterministic nature of the queueing models, we need to scale up the waiting times by $\sqrt{n}$ in order to get a nondegenerate limit in case (i). That is in stark contrast with (3.4), where we had to scale down the waiting times by $\sqrt{n}$. For the first case, the stationary waiting times were treated previously in [7] for the special case of the $\mathrm{GI}_{n} / \mathrm{D} / 1$ model, which has the GI/D/1 base model. In case (ii), even with the more rapid increase of $\rho_{n}$, we obtain a nondegenerate limit for the waiting times without any spatial scaling.

\subsection{Limits for scaled waiting times in case (i)}

We will express the HT limit for case (i) in terms of random elements of $\mathbb{R}^{\infty}$, using the discrete reflection map $\tilde{\phi}$ defined in (3.4). For that purpose, we introduce the following random elements of $\mathbb{R}^{\infty}$ : let

$$
\begin{gathered}
\tilde{S}_{n}^{c, u}(k) \equiv \sqrt{n}\left(S_{n, k}^{c, u}-k\right), \quad \tilde{S}_{n}^{c, v}(k) \equiv \sqrt{n}\left(S_{n, k}^{c, v}-k\right), \\
\tilde{S}_{n}^{c}(k) \equiv \sqrt{n} S_{n, k}^{c}, \quad \text { and } \quad \tilde{W}_{n}^{c}(k) \equiv \sqrt{n} W_{n, k}^{c}, \quad k \geq 1, n \geq 1,
\end{gathered}
$$


with $\tilde{S}_{n}^{c}(0) \equiv \sqrt{n} S_{n, 0} \equiv \sqrt{n} W_{n, 0}$, where $\left(S_{n, k}^{c, u}, S_{n, k}^{c, v}\right)$ is defined in (4.1), $S_{n, k}^{c} \equiv S_{n, k}^{c, v}-S_{n, k}^{c, u}$ and $W_{n, k}^{c}$ is defined in terms of $\left\{S_{n, k}^{c}: k \geq 0\right\}$ as in (3.1). The scaling in which we multiply by $\sqrt{n}$ converts the HT problem into a model continuity problem. When we considered HT limits for the stationary waiting times in [11], we applied the model continuity results in Section X.6 of [2] and Chapter 4 of [4].

Theorem 4.1. (HT limit for the scaled waiting times in the $\mathrm{G}_{n} / \mathrm{G}_{n} / 1$ models.) Consider $a$ sequence of $G_{n} / G_{n} / 1$ models associated with a base sequence of $G / G / 1$ models satisfying

$$
\left(\sqrt{n} W_{n, 0}, S_{n}^{u}, S_{n}^{v}\right) \Rightarrow\left(\tilde{W}(0), L^{u}, L^{v}\right) \quad \text { in } \mathbb{R} \times D^{2},
$$

where $\mathrm{P}\left(\left(L^{u}, L^{v}\right) \in C^{2}\right)=1$, as in Theorem 3.1, but the scaling of the initial conditions is changed. Then

$$
\left(\tilde{W}_{n}^{c}(0), \tilde{S}_{n}^{c, u}, \tilde{S}_{n}^{c, v}, \tilde{S}_{n}^{c}, \tilde{W}_{n}^{c}\right) \Rightarrow\left(\tilde{W}^{c}(0), \tilde{L}^{u}, \tilde{L}^{v}, \tilde{L}, \tilde{W}\right) \quad \text { in } \mathbb{R} \times\left(\mathbb{R}^{\infty}\right)^{3}
$$

where $\tilde{W} \equiv \tilde{\phi}(\tilde{W}(0)+\tilde{L})$ for $\tilde{\phi}$ defined in (3.4), $\tilde{L} \equiv \tilde{L}^{v}-\tilde{L}^{u}, \tilde{L}^{u}(k) \equiv L^{u}(k)$, and $\tilde{L}^{v}(k) \equiv$ $L^{v}(k), k \geq 1$, with $\left(L^{u}, L^{v}\right)$ from (3.7).

Proof. By (4.1) and (4.3),

$$
\left(\tilde{W}_{n}^{c}(0), \tilde{S}_{n}^{c, u}(k), \tilde{S}_{n}^{c, v}(k)\right)=\left(\sqrt{n} W_{n, 0}, S_{n}^{u}(k), S_{n}^{v}(k)\right) \quad \text { for } k \geq 1,
$$

where $\left(\boldsymbol{S}_{n}^{u}, \boldsymbol{S}_{n}^{v}\right)$ is the random element of $D^{2}$ defined in (3.5). By (4.4), $\left(\sqrt{n} W_{n, 0}, \boldsymbol{S}_{n}^{u}, \boldsymbol{S}_{n}^{v}\right) \Rightarrow$ $\left(\tilde{W}(0), L^{u}, L^{v}\right)$. Applying the continuous mapping theorem with the projection map for the last two components, $\pi_{1,2, \ldots, k}: \mathbb{R} \times D^{2} \rightarrow \mathbb{R} \times\left(\mathbb{R}^{2}\right)^{k} \equiv \mathbb{R}^{2 k+1}$, defined by $\pi_{1,2, \ldots, k}(a, x) \equiv$ $(a, x(1), x(2), \ldots x(k))$, we deduce convergence on the initial segments, which implies convergence of the first three components of (4.5) in $\mathbb{R} \times\left(\mathbb{R}^{\infty}\right)^{2}$. We apply the continuous mapping theorem again with addition and discrete reflection to treat the final two components.

If we impose all the additional conditions in Theorem 3.2, then we obtain a reflected Gaussian random walk as a limit; we treat the stationary distributions in [11].

Corollary 4.1. (HT limit for the scaled waiting times in standard $\mathrm{G}_{n} / \mathrm{G}_{n} / 1$ models.) Consider a sequence of $G_{n} / G_{n} / 1$ models associated with a base sequence of $G / G / 1$ models satisfying

$$
\left(\sqrt{n} W_{n, 0}, \hat{\boldsymbol{S}}_{n}^{u}, \hat{\boldsymbol{S}}_{n}^{v}\right) \Rightarrow\left(\tilde{W}(0), \hat{L}^{u}, \hat{L}^{v}\right) \quad \text { in } \mathbb{R} \times D^{2}
$$

for $\left(\hat{\boldsymbol{S}}_{n}^{u}, \hat{\boldsymbol{S}}_{n}^{v}\right)$ in (3.9), where $\mathrm{P}\left(\left(\hat{L}^{u}, \hat{L}^{v}\right) \in C^{2}\right)=1$. If $\left(1-\rho_{n}\right) \sqrt{n} \rightarrow \beta, 0<\beta<\infty$, as $n \rightarrow \infty$, as in (3.12), then the limit in (4.5) holds with $L^{u}=\hat{L}^{u}+\beta e$ and $L^{v}=\hat{L}^{v}$. If, in addition, condition (3.14) of Theorem 3.2 holds then $L=\sigma B-\beta e$, where $B$ is a standard $B M$ and $e$ is the identity map in $D$, so that $\tilde{W}$ becomes a reflected Gaussian random walk with negative drift, starting at the independent initial state $\tilde{W}(0)$; in particular,

$$
\tilde{W} \equiv\{\tilde{W}(k): k \geq 0\}=\{\tilde{\phi}(\tilde{W}(0)+\sigma B-\beta e)(k): k \geq 1\} \quad \text { in } \mathbb{R}^{\infty} .
$$

\subsection{Limits for unscaled waiting times in case (ii)}

We now obtain a different limit in case (ii). Here it will be convenient to exploit the singlesequence framework of Section 3. In this case, we express the HT limit in terms of random 
elements of $D$. For that purpose, let

$$
\begin{aligned}
S_{n}^{c, u}(t) & \equiv S_{n,\lfloor n t\rfloor}^{c, u}-\lfloor n t\rfloor=\frac{S_{n\lfloor n t\rfloor}^{u}-\lfloor n t\rfloor,}{\rho_{n} n} \\
S_{n}^{c, v}(t) & \equiv S_{n,\lfloor n t\rfloor}^{c, v}-\lfloor n t\rfloor=\frac{S_{n, n\lfloor n t\rfloor}^{v}-n\lfloor n t\rfloor}{n}=\frac{S_{n\lfloor n t\rfloor}^{v}-n\lfloor n t\rfloor}{n}, \\
S_{n}^{c}(t) & \equiv S_{n,\lfloor n t\rfloor}^{c}=\frac{S_{n, n\lfloor n t\rfloor}^{v}}{n}-\frac{S_{n, n\lfloor n t\rfloor}^{u}}{\rho_{n} n}=\left(S_{n}^{c, v}-S_{n}^{c, u}\right)(t), \\
\boldsymbol{W}_{n}^{c}(t) & \equiv W_{n,\lfloor n t\rfloor}^{c}=\frac{W_{n, n\lfloor n t\rfloor}}{n}=\phi\left(\boldsymbol{W}_{n}^{c}(0)+\boldsymbol{S}_{n}^{c}\right)(t) .
\end{aligned}
$$

Theorem 4.2. (HT limit for the unscaled waiting times in the $\mathrm{G}_{n} / \mathrm{G}_{n} / 1$ models.) Consider a sequence of $G_{n} / G_{n} / 1$ models associated with a single base $G / G / 1$ model satisfying

$$
\left(W_{n, 0}, \hat{\boldsymbol{S}}_{n}^{u}, \hat{\boldsymbol{S}}_{n}^{v}\right) \Rightarrow\left(\boldsymbol{W}^{c}(0), \hat{L}^{u}, \hat{L}^{v}\right) \quad \text { in } \mathbb{R} \times D^{2}
$$

for $\left(\hat{\boldsymbol{S}}_{n}^{u}, \hat{\boldsymbol{S}}_{n}^{v}\right)$ in (3.9), where $\mathrm{P}\left(\left(\hat{L}^{u}, \hat{L}^{v}\right) \in C^{2}\right)=1$ (just as in (3.11) except for the initial conditions). Instead of condition (3.12) of Theorem 3.2, assume that

$$
\left(1-\rho_{n}\right) n \rightarrow \beta, \quad 0<\beta<\infty, \text { as } n \rightarrow \infty,
$$

as in case (ii). Then, as $n \rightarrow \infty$,

$$
\left(\boldsymbol{S}_{n}^{c}(0), \boldsymbol{S}_{n}^{c, u}, \boldsymbol{S}_{n}^{c, v}, \boldsymbol{S}_{n}^{c}, \boldsymbol{W}_{n}^{c}\right) \Rightarrow\left(\boldsymbol{W}^{c}(0), \hat{L}^{u}+\beta e, \hat{L}^{v}, L, \boldsymbol{W}^{c}\right) \quad \text { in } \mathbb{R} \times D^{4},
$$

where $\boldsymbol{W}^{c} \equiv \phi\left(\boldsymbol{W}^{c}(0)+L\right), \phi$ is given in (3.6), $L \equiv \hat{L}^{v}-\hat{L}^{u}-\beta e$, and $\left(\hat{L}^{u}, \hat{L}^{v}\right)$ comes from (3.11). If, in addition condition (3.14) of Theorem 3.2 holds then $L \stackrel{D}{=} \sigma B-\beta e$, where $B$ is a standard $B M$ and $\sigma^{2}=\sigma_{u}^{2}+\sigma_{v}^{2}$.

Proof. We are exploiting the single-sequence framework in Section 3.2, because the final expressions in (4.6a) and (4.6b) involve only the single sequence. We will relate our processes $\left(\boldsymbol{S}_{n}^{c, u}, \boldsymbol{S}_{n}^{c, v}\right)$ directly to these. From the formulae in (4.6), we see that $\boldsymbol{S}_{n}^{c, v}(t)=\hat{\boldsymbol{S}}_{n^{2}}^{v}(t)$ for $t=$ $k / n$ for all nonnegative integers $n$ and $k$, while $S_{n}^{c, v}$ is constant in all intervals $[k / n,(k+1) / n)$. Now let $\|\cdot\|_{t}$ be the uniform norm for $\mathbb{R}^{k}$-valued functions on the interval $[0, t]$, using the maximum norm $|\cdot|$ in $\mathbb{R}^{k}$, and let the modulus of continuity be defined for any $x \in D$ by

$$
w_{x}(\delta, t) \equiv \sup \left\{\left|x\left(t_{1}\right)-x\left(t_{2}\right)\right|: 0 \leq t_{1}<t_{2} \leq t,\left|t_{2}-t_{1}\right|<\delta\right\} .
$$

Hence,

$$
\left\|S_{n}^{c, v}-S_{n^{2}}^{v}\right\|_{t} \leq w_{S_{n^{2}}^{v}}\left(\frac{1}{n}, t\right) \text { for each } n \geq 1 \text { and } t>0 .
$$

By (3.11) and the fact that $\mathrm{P}\left(\hat{L}^{v} \in C^{2}\right)=1$, we have $w_{\hat{S}_{n^{2}}^{v}}(1 / n, t) \Rightarrow 0$ as $n \rightarrow \infty$, which with (4.9) implies that

$$
\left\|\boldsymbol{S}_{n}^{c, v}-\hat{\boldsymbol{S}}_{n^{2}}^{v}\right\|_{t} \rightarrow 0 \quad \text { as } n \rightarrow \infty .
$$

A minor modification of the same reasoning applies to $\boldsymbol{S}_{n}^{c, u}(t)$. We need to be more careful because of the extra scaling by $\rho_{n}$. For that purpose, we introduce the fluid scaled process

$$
\bar{S}_{n}^{u}(t) \equiv \frac{S_{\lfloor n t\rfloor}^{u}}{n}, \quad t \geq 0 .
$$


Given the assumed limit in (3.11), we have the extension $\left(\hat{\boldsymbol{S}}_{n}^{u}, \overline{\boldsymbol{S}}_{n}^{u}, \hat{\boldsymbol{S}}_{n}^{v}\right) \Rightarrow\left(\hat{L}^{u}, e, \hat{L}^{v}\right)$ in $D^{3}$. Now, using the reasoning in (3.15) and (3.16), we observe that

$$
\boldsymbol{S}_{n}^{c, u}(t)=Z_{n^{2}}(t) \equiv \hat{\boldsymbol{S}}_{n^{2}}^{u}(t)+(\beta+o(1)) \overline{\boldsymbol{S}}_{n^{2}}^{u}(t)
$$

for $t=k / n$ for all integers $k \geq 0$ and $n \geq 1$, while $S_{n}^{c, u}$ remains constant in each interval $[k / n,(k+1) / n)$. Hence, reasoning as for $\boldsymbol{S}_{n}^{c, v}$ above, we have

$$
\left\|S_{n}^{c, u}-Z_{n^{2}}\right\|_{t} \leq w_{Z_{n^{2}}}\left(\frac{1}{n}, t\right) \text { for each } n \geq 1 \text { and } t>0 .
$$

Since $Z_{n} \Rightarrow \hat{L}^{u}+\beta e$, where $\mathrm{P}\left(\hat{L}^{u}+\beta e \in C\right)=1$, we have $w_{Z_{n^{2}}}(1 / n, t) \Rightarrow 0$ as $n \rightarrow \infty$ for all $t>0$. Combining the results above, we have

$$
\left\|\left(W_{n, 0}, S_{n}^{c, u}, S_{n}^{c, v}\right)-\left(W_{n, 0}, Z_{n^{2}}, S_{n^{2}}^{v}\right)\right\| \Rightarrow 0 .
$$

That in turn, with the 'convergence-together theorem', Theorem 11.4.7 of [14], implies convergence for the first three terms in (4.8). Finally, we apply the continuous mapping theorem with subtraction and reflection to get the full limit in (4.8).

Remark 4.1. (Practical significance of the scaling.) We want to emphasize the practical significance of the scaling of space and time in Theorem 4.2. To do so, it is helpful to focus on a single system with traffic intensity $\rho$, which we can relate to $n$ by replacing the assumed growth condition by an equality. For the original G/G/1 model, with $(1-\rho) \sqrt{n} \rightarrow \beta$, we have $n^{-1 / 2} W_{n,\lfloor n t\rfloor} \Rightarrow \phi(L)(t)$. Hence, letting $(1-\rho) \sqrt{n}=\beta=1$, we see that, for higher values of $\rho, W_{k}^{\rho}$ should be of order $O(1 /(1-\rho))$, while significant changes occur over time intervals of length $O\left(1 /\left(1-\rho^{2}\right)\right)$.

In contrast, with cyclic thinning, we have the alternative growth condition $(1-\rho) n \rightarrow \beta$ in (4.7), under which $W_{n,\lfloor n t\rfloor}^{c} \Rightarrow \phi(L)(t)$. Now we can let $\left(1-\rho_{n}\right) n=\beta=1$ and obtain $n=1 /(1-\rho)$. With cyclic thinning, we have $W_{k}^{c, \rho}$ being of order $O(1)$, while significant changes occur over a time scale of $O(1 /(1-\rho))$.

The time scaling by $n$ with cyclic thinning can be better understood by considering the approximating $\mathrm{D} / \mathrm{D} / 1$ model with the given traffic intensity. For example, suppose that the service times are all 1 and the interarrival times are all $n /(n-1)$ for some large positive integer $n$, corresponding to $\left(1-\rho_{n}\right) n=1$. Suppose that the system is initialized by an arrival at time 0 who finds $k$ customers in the queue and one more in service at the beginning of a service time. Because of the deterministic service times, that initial customer at time 0 has a waiting time of exactly $k+1$. In general, if there are $k$ customers in the queue upon arrival, the waiting time is bounded below by $k$ and bounded above by $k+1$. Thus, in the $\mathrm{D} / \mathrm{D} / 1$ model, the waiting time is tightly linked to the queue length. For the specified initial conditions, it takes time $n$ for the queue length to decrease by 1 ; the queue will first become empty, leaving one customer in service just beginning his/her service time, at time $k n$. Thus, the waiting time of a new arrival at time $j n$ will be about $k+1-j$. Thus, we see that in the $\mathrm{D} / \mathrm{D} / 1$ model with alternative initial conditions the waiting times change over time periods of order $n$. Theorem 4.2 is showing that remains true in the HT limit for the $\mathrm{G}_{n} / \mathrm{G}_{n} / 1$ model. Finally, the extra variability in the $\mathrm{G}_{n} / \mathrm{G}_{n} / 1$ model produces the stochastic limit without spatial scaling in Theorem 4.2.

To highlight Remark 4.1, we state a corollary for first passage times, obtained by applying the continuous mapping theorem with the limit in (4.8). Let $a$ and $b$ be real numbers with 
$0<a<b<\infty$. Consider $x \in D$ with $x(0)=c$ for $c$ to be specified. Then define the first passage functions

$$
T_{a, b}^{+}(x) \equiv \inf \{t \geq 0: x(t)>b \mid x(0)=a\}
$$

and $T_{b, a}^{-}(x) \equiv \inf \{t \geq 0: x(t)<a \mid x(0)=b\}$. We will be interested in

$$
T_{a, b}^{+}\left(\boldsymbol{W}_{n}^{c}\right)=n^{-1} \min \left\{k \geq 1: W_{n, k}^{c}>b \mid W_{n, 0}^{c}=a\right\}
$$

and $T_{b, a}^{-}\left(\boldsymbol{W}_{n}^{c}\right)=n^{-1} \min \left\{k \geq 1: W_{n, k}^{c}<a: W_{n, 0}^{c}=b\right\}$.

For RBM and the M/M/1 queue, the distributions of the first passage times $T_{0, x}$ and $T_{x, 0}$ are described in Section 5.7.5 of [14]. There the limits are described in terms of canonical BM and RBM with negative drift, having drift rate -1 and diffusion coefficient 1 , which is convenient, because there are no parameters. It is easy to transform BM and RBM with general parameters to and from the canonical versions; see page 174 of [14].

Corollary 4.2. (HT limit for first passage times in the $\mathrm{G}_{n} / \mathrm{G}_{n} / 1$ models.) Under the assumptions of Theorem 4.2, including condition (3.14) of Theorem 3.2,

$$
\begin{aligned}
n^{-1} \min \left\{k \geq 1: W_{n, k}^{c}>b \mid W_{n, 0}^{c}=a\right\} & \Rightarrow T_{a, b}^{+}(\phi(\sigma B-\beta e)) \\
& \stackrel{\mathrm{D}}{=} \frac{\sigma^{2}}{\beta^{2}} T_{\beta a / \sigma^{2}, \beta b / \sigma^{2}}^{+}(\phi(B-e)), \\
n^{-1} \min \left\{k \geq 1: W_{n, k}^{c}<a \mid W_{n, 0}^{c}=b\right\} & \Rightarrow T_{b, a}^{-}(\phi(\sigma B-\beta e)) \\
& \stackrel{\mathrm{D}}{=} T_{b, a}^{-}(\sigma B-\beta e) \\
& \stackrel{\mathrm{D}}{=} \frac{\sigma^{2}}{\beta^{2}} T_{\beta b / \sigma^{2}, \beta a / \sigma^{2}}^{-}(B-e) .
\end{aligned}
$$

For example, since $\mathrm{E}\left[T_{b, a}^{-}(B-e)\right]=\operatorname{var}\left(T_{b, a}^{-}(B-e)\right)=b-a$, we have

$$
\begin{aligned}
\mathrm{E}\left[\frac{\sigma^{2}}{\beta^{2}} T_{\beta b / \sigma^{2}, \beta a / \sigma^{2}}^{-}(B-e)\right] & =\frac{b-a}{\beta}, \\
\operatorname{var}\left(\frac{\sigma^{2}}{\beta^{2}}\right) T_{\beta b / \sigma^{2}, \beta a / \sigma^{2}}^{-}(B-e) & =\frac{\sigma^{2}(b-a)}{\beta^{3}} .
\end{aligned}
$$

\section{Associated counting processes}

Theorem 4.2 shows that the individual partial sums $S_{n, k}^{c, u}$ and $S_{n, k}^{c, v}$ in (4.1), associated with the arrival process and service processes obtained from cyclic thinning, satisfy FCLTs with appropriate scaling, because the sequence of random elements $\left\{\left(\boldsymbol{S}_{n}^{c, u}, \boldsymbol{S}_{n}^{c, v}\right): n \geq 1\right\}$ in (4.6) is asymptotically equivalent to the associated sequence of random elements $\left\{\left(\hat{\boldsymbol{S}}_{n^{2}}^{u}+\right.\right.$ $\left.\left.\beta \overline{\boldsymbol{S}}_{n^{2}}^{u}, \hat{\boldsymbol{S}}_{n^{2}}^{v}\right): n \geq 1\right\}$ in (3.9) and (4.10) with the scaling in (3.10) as $n \rightarrow \infty$, i.e. because of (4.11) and (4.12).

We would thus naturally expect that FCLTs would hold for the associated counting processes, by virtue of the continuous mapping theorem applied with the inverse function, as in Sections 13.6-13.8 of [14]. However, that is not so. Upon closer examination, we find that the asymptotically negligible differences between $\left(\boldsymbol{S}_{n}^{c, u}, \boldsymbol{S}_{n}^{c, v}\right)$ and $\left(\hat{\boldsymbol{S}}_{n^{2}}^{u}+\beta \overline{\boldsymbol{S}}_{n^{2}}^{u}, \hat{\boldsymbol{S}}_{n^{2}}^{v}\right)$ significantly affect the associated counting processes. Nevertheless, we do establish FCLTs for the counting processes with different scaling. 
To treat the associated counting processes, let

$$
\begin{aligned}
N_{n}^{u}(t) & \equiv \max \left\{k \geq 0: S_{n, k}^{u} \leq t\right\}, & & N_{n}^{v}(t) \equiv \max \left\{k \geq 0: S_{n, k}^{v} \leq t\right\}, \\
N_{n}^{c, u}(t) & \equiv \max \left\{k \geq 0: S_{n, k}^{c, u} \leq t\right\}, & & N_{n}^{c, v}(t) \equiv \max \left\{k \geq 0: S_{n, k}^{c, v} \leq t\right\},
\end{aligned}
$$

for $t \geq 0$. For simplicity, now assume in addition that $\mathrm{P}\left(U_{n, k}>0\right)=1$ and $\mathrm{P}\left(V_{n, k}>0\right)=1$ for all $n$ and $k$, so that all these counting processes increase by unit jumps. By our initial conditions in Section 3.1, we have $N_{n}^{u}(0)=1$ and $N_{n}^{v}(0)=0$.

\subsection{No time scaling}

Before observing the technical problems associated with the counting processes if we use the scaling in Theorem 4.2, we observe that the time scaling there by $n$ plays a critical role in obtaining interesting nondegenerate stochastic limits. In particular, we now show that if we do not scale time by $n$ in the $\mathrm{G}_{n} / \mathrm{G}_{n} / 1$ models as $n \rightarrow \infty$ under the conditions of Theorem 4.2 , we simply get convergence of all these queueing processes to the associated deterministic processes in the trivial D/D/1 model with traffic intensity 1 (and the specified initial conditions, having a 0th customer arrive at time 0 to find an empty system). (The results in this subsection hold for both $\left(1-\rho_{n}\right) n \rightarrow \beta$ as in (4.7) or $\left(1-\rho_{n}\right) \sqrt{n} \rightarrow \beta$ as in (3.12).)

We start by considering the counting processes. For the counting processes, when we combine (5.1) with the basic definition in (4.1) and the initial conditions in Section 3.1, we obtain the important relations

$$
N_{n}^{u}(n t)=1+n\left(N_{n}^{c, u}(t)-1\right)+J_{n}^{c, u}(t) \quad \text { and } \quad N_{n}^{v}(n t)=n N_{n}^{c, v}(t)+J_{n}^{c, v}(t),
$$

where $J_{n}^{c, u}(t)$ counts the number of interarrival time phases completed in the interarrival time in progress at time $t$, while $J_{n}^{c, v}(t)$ counts the number of service time phases completed in the service time in progress at time $t$. By our assumed initial conditions, $J_{n}^{c, u}(0)=J_{n}^{c, v}(0)=0$ for all $n \geq 1$. Clearly, $0 \leq J_{n}^{c, u}(t)<n$ and $0 \leq J_{n}^{c, v}(t)<n$ for all $t \geq 0$ and $n \geq 1$. We can then rewrite the relations in (5.2) as

$$
\begin{aligned}
& N_{n}^{c, u}(t)=1+\frac{N_{n}^{u}(n t)-1}{n}-\frac{J_{n}^{c, u}(t)}{n}=1+\left\lfloor\frac{\left.N_{n}^{u}(n t)-1\right)}{n}\right\rfloor, \\
& N_{n}^{c, v}(t)=\frac{N_{n}^{v}(n t)}{n}-\frac{J_{n}^{c, v}(t)}{n}=\left\lfloor\frac{N_{n}^{v}(n t)}{n}\right\rfloor,
\end{aligned}
$$

where $\lfloor t\rfloor$ is again the floor function, which is right continuous and, thus, an element of $D$.

Given the spatial scaling on the right-hand side of (5.3), we can obtain an FWLLN for $\left(N_{n}^{c, u}, N_{n}^{c, v}\right)$, but only in the product space $D^{2}$. We cannot obtain convergence in $D\left([0, \infty), \mathbb{R}^{2}\right)$ with the usual $J_{1}$ topology, because the limit functions have common discontinuity points, and we require inconsistent time transformations in the two components. Convergence of the components separately is easy, because all processes are integer valued. Hence, convergence in $D$ is equivalent to convergence of the finite-dimensional distributions. To state the results, first introduce the FWLLN-scaled processes associated with (3.9):

$$
\overline{\boldsymbol{S}}_{n}^{u}(t) \equiv \frac{S_{\lfloor n t\rfloor}^{u}}{n} \quad \text { and } \quad \overline{\boldsymbol{S}}_{n}^{v}(t) \equiv \frac{S_{\lfloor n t\rfloor}^{v}}{n}, \quad t \geq 0 .
$$

Next define the following random elements in $D$ :

$$
\overline{\boldsymbol{N}}_{n}^{u}(t) \equiv \frac{N_{n}^{u}(n t)}{n}, \quad \overline{\boldsymbol{N}}_{n}^{v}(t) \equiv \frac{N_{n}^{v}(n t)}{n}, \quad \overline{\boldsymbol{J}}_{n}^{c, u} \equiv \frac{J_{n}^{c, u}(t)}{n}, \quad \overline{\boldsymbol{J}}_{n}^{c, v} \equiv \frac{J_{n}^{c, v}(t)}{n}
$$


In (5.5) and in Theorem 5.1 below, we have no time scaling by $n$ for the processes associated with the $\mathrm{G}_{n} / \mathrm{G}_{n} / 1$ model, whereas we do for the associated $\mathrm{G} / \mathrm{G} / 1$ base model.

Theorem 5.1. (FWLLN for the counting processes with cyclic thinning.) Consider a sequence of $G_{n} / G_{n} / 1$ models associated with a single base $G / G / 1$ model satisfying

$$
\left(\overline{\boldsymbol{S}}_{n}^{u}, \overline{\boldsymbol{S}}_{n}^{v}\right) \Rightarrow(e, e) \text { in } D^{2}
$$

for $\left(\overline{\boldsymbol{S}}_{n}^{u}, \overline{\boldsymbol{S}}_{n}^{v}\right)$ in (5.4). If either (4.7) or (3.12) holds, then

$$
\left(\overline{\boldsymbol{N}}_{n}^{u}, \overline{\boldsymbol{J}}_{n}^{c, u}, N_{n}^{c, u}, \overline{\boldsymbol{N}}_{n}^{v}, \overline{\boldsymbol{J}}_{n}^{c, v}, N_{n}^{c, v}\right) \Rightarrow(e, J, 1+\lfloor e\rfloor, e, J,\lfloor e\rfloor) \quad \text { in } D^{6} \text { as } n \rightarrow \infty,
$$

where $e$ is the identity map in $D,\lfloor e\rfloor(t) \equiv\lfloor t\rfloor$, and $J=e-\lfloor e\rfloor$.

Proof. Assume that (4.7) holds; the reasoning is similar with (3.12). We get the limit for the first and fourth terms by applying the continuous mapping theorem with the inverse map, i.e. Theorem 13.6.1 of [14]. Moreover, by (5.3), we can write

$$
N_{n}^{c, v}=\left\lfloor\bar{N}_{n}^{v}\right\rfloor \quad \text { for all } n \quad \text { and } \quad d_{t}\left(N_{n}^{c, u}, 1+\left\lfloor\bar{N}_{n}^{u}\right\rfloor\right) \Rightarrow 0
$$

as $n \rightarrow \infty$ for all noninteger $t>0$, where $d_{t}$ is a metric inducing the $J_{1}$ topology on $D([0, t])$. Hence, we also have $\left(N_{n}^{c, u}, N_{n}^{c, v}\right) \Rightarrow(1+\lfloor e\rfloor,\lfloor e\rfloor)$ in $D^{2}$. Finally, we also have $\left(\overline{\boldsymbol{J}}_{n}^{c, u}, \overline{\boldsymbol{J}}_{n}^{c, v}\right) \Rightarrow(J, J)$ in $D^{2}$ as $n \rightarrow \infty$ from the above and (5.3). This completes the proof.

\subsection{Time-scaled counting processes}

The story is different if we scale time by $n$, as we have already seen in Theorem 4.2. In particular, the queue length and workload processes in the $\mathrm{G}_{n} / \mathrm{G}_{n} / 1$ model for large $n$ will fluctuate randomly over time intervals of length $O(n)$. We first consider the FCLT refinement of the FWLLN in Theorem 5.1. We get the following result, which is partly positive and partly negative. To state the result, in addition to the processes in (5.12) below, we introduce the following random elements for each $n \geq 1$ :

$$
\begin{gathered}
N_{n}^{c, u} \equiv N_{n}^{c, u}(n t)-n t, \quad N_{n}^{c, v} \equiv N_{n}^{c, v}(n t)-n t, \\
\boldsymbol{J}_{n}^{c, u} \equiv \frac{J_{n}^{c, u}(n t)}{n}, \quad \text { and } \quad \boldsymbol{J}_{n}^{c, v} \equiv \frac{J_{n}^{c, v}(n t)}{n},
\end{gathered}
$$

for $t \geq 0$. Paralleling (3.5) and (4.6), in (5.7) the processes in $\left(\boldsymbol{N}_{n}^{u}, \boldsymbol{N}_{n}^{u}, \boldsymbol{J}_{n}^{c, u}, \boldsymbol{J}_{n}^{c, v}\right)$ have spatial scaling, but the processes in $\left(\boldsymbol{N}_{n}^{c, u}, \boldsymbol{N}_{n}^{c, u}\right)$ do not. To state the result, let ' $\leq_{\mathrm{st}}$ ' denote the ordinary stochastic order for real-valued random variables, i.e. we write $X_{1} \leq_{\text {st }} X_{2}$ for real-valued random variables if $\mathrm{P}\left(X_{1}>t\right) \leq \mathrm{P}\left(X_{2}>t\right)$ for all $t$.

Theorem 5.2. (FCLT for the counting processes with cyclic thinning.) Consider a sequence of $G_{n} / G_{n} / 1$ models associated with a single base $G / G / 1$ model satisfying

$$
\left(\hat{\boldsymbol{S}}_{n}^{u}, \hat{\boldsymbol{S}}_{n}^{v}\right) \Rightarrow\left(\hat{L}^{u}, \hat{L}^{v}\right) \text { in } D^{2}
$$

for $\left(\hat{\boldsymbol{S}}_{n}^{u}, \hat{\boldsymbol{S}}_{n}^{v}\right)$ in (3.9), where $\mathrm{P}\left(\left(\hat{L}^{u}, \hat{L}^{v}\right) \in C^{2}\right)=1$. Assume that either (4.7) or (3.12) holds. Then

$$
\left(\boldsymbol{N}_{n}^{c, u}+\boldsymbol{J}_{n}^{c, u}, \boldsymbol{N}_{n}^{c, v}+\boldsymbol{J}_{n}^{c, u}\right) \Rightarrow\left(1-\hat{L}^{u}-\beta e,-\hat{L}^{v}\right) \quad \text { in } D^{2} \text { as } n \rightarrow \infty,
$$

where $\left(\hat{L}^{u}, \hat{L}^{v}\right)$ comes from (3.11). However, the sequences $\left\{\boldsymbol{J}_{n}^{c, u}: n \geq 1\right\},\left\{\boldsymbol{J}_{n}^{c, v}: n \geq 1\right\}$, $\left\{N_{n}^{c, u}: n \geq 1\right\}$, and $\left\{N_{n}^{c, v}: n \geq 1\right\}$ in $D$ are not tight and, thus, do not converge in $D$. 
Nevertheless, for each $t \geq 0$, the associated sequences of real-valued random variables $\left\{\boldsymbol{J}_{n}^{c, u}(t): n \geq 1\right\},\left\{\boldsymbol{J}_{n}^{c, v}(t): n \geq 1\right\},\left\{\boldsymbol{N}_{n}^{c, u}(t): n \geq 1\right\}$, and $\left\{\boldsymbol{N}_{n}^{c, v}(t): n \geq 1\right\}$ are tight. Moreover, for any convergent subsequence, with limits denoted by $\boldsymbol{J}^{c, u}(t), \boldsymbol{J}^{c, v}(t), \boldsymbol{N}^{c, u}(t)$, and $N^{c, v}(t)$, we have the following bounds:

$$
\begin{aligned}
& \mathrm{P}\left(0 \leq \boldsymbol{J}^{c, u}(t) \leq 1\right)=\mathrm{P}\left(0 \leq \boldsymbol{J}^{c, v}(t) \leq 1\right)=1, \\
& -\hat{L}^{u}(t)-\beta t \leq_{\mathrm{st}} \boldsymbol{N}^{c, u}(t) \leq_{\mathrm{st}} 1-\hat{L}^{u}(t)-\beta t, \\
& -\hat{L}^{v}(t)-1 \leq_{\mathrm{st}} N^{c, v}(t) \leq_{\mathrm{st}}-\hat{L}^{v}(t) .
\end{aligned}
$$

Proof. From the basic FCLT equivalence for partial sums and counting processes expressed in Theorem 7.3.2 of [14], we obtain

$$
\left(\hat{N}_{n}^{u}, \hat{N}_{n}^{v}\right) \Rightarrow\left(-\hat{L}^{u},-\hat{L}^{v}\right) \text { in } D^{2} \text { as } n \rightarrow \infty,
$$

where

$$
\hat{N}_{n}^{u}(t) \equiv \frac{N^{u}(n t)-n t}{\sqrt{n}} \quad \text { and } \quad \hat{N}_{n}^{v}(t) \equiv \frac{N^{v}(n t)-n t}{\sqrt{n}}, \quad t \geq 0 .
$$

By (5.3) and (5.7), we have

$$
\boldsymbol{N}_{n}^{c, v}+\boldsymbol{J}_{n}^{c, v}=\boldsymbol{N}_{n^{2}}^{v} \quad \text { and } \quad\left\|\left(\boldsymbol{N}_{n}^{c, u}-1+\boldsymbol{J}_{n}^{c, u}\right)-\boldsymbol{N}_{n^{2}}^{u}\right\|_{t}=\frac{1}{n}
$$

for all $n$ and $t$, so that we can apply (5.11), which follows from the conditions, and the convergence-together theorem, Theorem 11.4.7 of [14], to obtain the positive result in (5.9). We use index $n^{2}$ instead of $n$.

We obtain the negative result by applying (5.13). We first observe that we have the established convergence of $\left(\boldsymbol{N}_{n^{2}}^{u}, \boldsymbol{N}_{n^{2}}^{v}\right)$ to a limit with continuous sample paths, as indicated in (5.11), so that

$$
\lim _{\delta \downarrow 0} \limsup _{n \rightarrow \infty} w_{N_{n^{2}}^{u}}(\delta, t)=0 \quad \text { and } \quad \lim _{\delta \downarrow 0} \limsup _{n \rightarrow \infty} w_{N_{n^{2}}^{v}}(\delta, t)=0 \quad \text { for all } t>0
$$

from the associated tightness. On the other hand, we have extra time scaling by $n$ to go from $\left(\overline{\boldsymbol{J}}_{n}^{c, u}, \overline{\boldsymbol{J}}_{n}^{c, v}\right)$ in (5.5) to $\left(\boldsymbol{J}_{n}^{c, u}, \boldsymbol{J}_{n}^{c, v}\right)$ in (5.7). Since $\left(\overline{\boldsymbol{J}}_{n}^{c, u}, \overline{\boldsymbol{J}}_{n}^{c, v}\right)$ converges to a nondegenerate limit with oscillations of size 1 over intervals of length 1 by Theorem 5.1, the oscillations will necessarily occur too rapidly as $n \rightarrow \infty$ when we add the extra time scaling by $n$. Hence, we cannot have tightness in $D$ for either $\boldsymbol{J}_{n}^{c, u}$ or $\boldsymbol{J}_{n}^{c, v}$, and so also for $\boldsymbol{N}_{n^{2}}^{u}-\boldsymbol{J}_{n}^{c, u}$ or $\boldsymbol{N}_{n^{2}}^{v}-\boldsymbol{J}_{n}^{c, v}$, which is necessary for convergence. However, from (5.13) we have stochastic bounds for each $n$, which does imply tightness of the associated sequence of real-valued random variables. Moreover, these stochastic bounds must be preserved under passing to the limit along any subsequence.

Under additional regularity conditions, we will have $\boldsymbol{J}_{n}^{c, u}(t)$ and $\boldsymbol{J}_{n}^{c, v}(t)$ converge to random variables with the uniform distribution on the interval $[0,1]$. Then we will be able to replace the bounds in (5.10) by convergence in distribution in $\mathbb{R}$. We obtained such stronger results in a stationary framework in [11].

We conclude this subsection by indicating a framework in which we can get a limit for $\left(\boldsymbol{J}_{n}^{c, u}, \boldsymbol{J}_{n}^{c, v}\right)$, but it is an unconventional limit, even going beyond the spaces $E$ and $F$ in Chapter 15 of [14]. We will make the underlying space a subset of the collection of compact 
subsets of $\mathbb{R}^{2}$. We put the sample paths of the stochastic processes in $D$ in this space by (i) looking at the graphs of the sample paths in $\mathbb{R}^{2}$, including the left limits, and (ii) by considering the restrictions of the domain $[0, \infty)$ to $[0, t]$ for various $t>0$. As in the conventional spaces $C$ and $D$, convergence will be characterized in terms of restrictions to the bounded interval $[0, t]$ for a sequence of time points $t$ converging to $\infty$. For background on this graph approach, see Chapters 12 and 15 of [14] and the references therein.

Instead of $D([0, t], \mathbb{R})$, we use the space $C_{t}$ of compact subsets of $[0, t] \times \mathbb{R}$ in $\mathbb{R}^{2}$ using the Hausdorff metric, denoted by $d_{\mathrm{H}, t}(A, B)$ and defined by

$$
d_{\mathrm{H}, t}(A, B) \equiv \max \left\{\sup _{x \in A} \inf _{y \in B} d(x, y), \sup _{y \in B} \inf _{x \in A} d(x, y)\right\},
$$

where $A, B \in C_{t}$ and $d$ is a metric on $\mathbb{R}^{2}$, for which it is convenient to take the maximum metric: $d(x, y) \equiv d\left(\left(x_{1}, x_{2}\right),\left(y_{1}, y_{2}\right)\right) \equiv \max \left\{\left|x_{1}-y_{1},\right| x_{2}-y_{2} \mid\right\}$; see of [14, p. 381] for background. For each $t,\left(\boldsymbol{C}_{t}, d_{\mathrm{H}, t}\right)$ is a compact metric space. Let $\boldsymbol{C}$ be the subset of $\mathbb{R}^{2}$ for which all restrictions to $[0, t] \times \mathbb{R}$ are compact, and let $\boldsymbol{C}^{k}$ be the $k$-fold product space with the product topology. Define convergence in $\boldsymbol{C}$ to mean convergence of the restrictions in $\boldsymbol{C}_{t}$ for all $t>0$. In our context, the limit of $\boldsymbol{J}_{n}^{c, u}$ is the deterministic set $\Upsilon \equiv[0, \infty) \times[0,1]$ in $\boldsymbol{C}$, which we call the unit blur. With this framework, we can obtain the following result.

Theorem 5.3. (Convergence to the unit blur.) Under the assumptions of Theorem 5.1,

$$
\left(\boldsymbol{J}_{n}^{c, u}, \boldsymbol{J}_{n}^{c, v}\right) \Rightarrow\left(\Upsilon^{u}, \Upsilon^{v}\right) \quad \text { in } \boldsymbol{C}^{2} \text { as } n \rightarrow \infty
$$

where $\Upsilon^{u}$ and $\Upsilon^{v}$ are unit blurs, so that

$$
\left(N_{n}^{c, u}, J_{n}^{c, u}, N_{n}^{c, v}, J_{n}^{c, u}\right) \Rightarrow\left(-\hat{L}^{u}-\beta e+\Upsilon^{u}, \Upsilon^{u},-\hat{L}^{v}-\Upsilon^{v}, \Upsilon^{v}\right) \quad \text { in } C^{4} \text { as } n \rightarrow \infty
$$

Proof. The statement in (5.14) is equivalent to convergence of the components separately, since the limit is deterministic, by Theorem 11.4.5 of [14]. First, for (5.14), we can exploit the FWLLN, Theorem 5.1. By (5.6),

$$
\left(\overline{\boldsymbol{J}}_{n}^{c, u}, \overline{\boldsymbol{J}}_{n}^{c, v}\right) \Rightarrow(J, J) \quad \text { in } D^{2} \text { as } n \rightarrow \infty
$$

where $J(t) \equiv t-\lfloor t\rfloor, t \geq 0$. For $x \in D$ and any constant $b>0$, let $(x \circ b e)(t) \equiv x(b t), t \geq 0$. From (5.16), we get the associated limit

$$
\left(\overline{\boldsymbol{J}}_{n}^{c, u} \circ b e, \overline{\boldsymbol{J}}_{n}^{c, v} \circ b e\right) \Rightarrow(J \circ b e, J \circ b e) \quad \text { in } D^{2} \text { as } n \rightarrow \infty
$$

for any $b>0$. We now carry out the remaining analysis only for the arrival process; the service process is treated in the same way.

First, we observe that, for any $\varepsilon>0$, we can choose $b_{\varepsilon}$ such that

$$
d_{\mathrm{H}, t}\left(J \circ b e, \Upsilon^{u}\right)<\varepsilon \quad \text { for all } b>b_{\varepsilon} \text { with probability } 1 .
$$

Next observe that

$$
d_{\mathrm{H}, t}\left(\left(\boldsymbol{J}_{n}^{c, u}, \Upsilon^{u}\right) \leq \sup _{b>n} d_{\mathrm{H}, t}\left(\overline{\boldsymbol{J}}_{n}^{c, u} \circ b e, \Upsilon^{u}\right)\right.
$$

where

$$
d_{\mathrm{H}, t}\left(\overline{\boldsymbol{J}}_{n}^{c, u} \circ b e, \Upsilon^{u}\right) \leq d_{\mathrm{H}, t}\left(\overline{\boldsymbol{J}}_{n}^{c, u} \circ b e, J \circ b e\right)+d_{\mathrm{H}, t}\left(J \circ b e, \Upsilon^{u}\right)
$$


For any given $\varepsilon>0$, by (5.18), we can make the second term on the right-hand side of (5.19) less than $\varepsilon / 2$ by choosing $b$ large enough. For that $b$ chosen, by (5.17) we can make the first term on the right-hand side of (5.19) less than $\varepsilon / 2$ by choosing $n$ large enough.

Finally, to establish (5.15), we use the fact that the convergence of $\left(\boldsymbol{N}_{n}^{c, u}+\boldsymbol{J}_{n}^{c, u}, \boldsymbol{N}_{n}^{c, v}+\boldsymbol{J}_{n}^{c, u}\right)$ established in $D^{2}$ implies the corresponding weaker convergence in $\boldsymbol{C}^{2}$ to the limit, which has single-valued projections at $t$ for all $t$. Since one of the limits has a single-valued projection, subtraction is continuous. The first component on the right-hand side of (5.15) is initially $1-\hat{L}^{u}-\beta e-\Upsilon$, but $1-\Upsilon=\Upsilon$.

Remark 5.1. (Interpreting the blur limits.) We note that we do not obtain the information about the joint distribution of $\Upsilon^{u}$ and $\Upsilon^{v}$. In particular, we cannot conclude that $\left(\Upsilon^{u}, \Upsilon^{v}\right)=$ $[0, \infty) \times[0,1] \times[0,1]$, because we do not know when each function assumes the values in $[0,1]$. In the framework above, $\Upsilon^{u}(t)=\Upsilon^{v}(t)=[0,1]$ for all $t \geq 0$, where $\Upsilon(t)$ is the projection of $\Upsilon$ on $t$. However, if we make additional stationarity and independence assumptions, we will have

$$
\left(\boldsymbol{J}_{n}^{c, u}(t), \boldsymbol{J}_{n}^{c, v}(t)\right) \stackrel{\mathrm{D}}{=}\left(Y_{n}^{u}, Y_{n}^{v}\right) \Rightarrow\left(Y^{u}, Y^{v}\right) \text { in } \mathbb{R}^{2},
$$

where $Y_{n}^{u}$ and $Y_{n}^{v}$ are independent random variables uniformly distributed on the finite set $\{0,1 / n, \ldots,(n-1) / n\}$, while $Y^{u}$ and $Y^{v}$ are independent random variables uniformly distributed on $[0,1]$. Then we would have the limit at $t$ for $\boldsymbol{J}_{n}^{c, u}(t)$ uniformly distributed over $[0,1]$, but our framework does not provide that extra level of detail. On the other hand, our framework has the virtue that it shows that, asymptotically as $n \rightarrow \infty$, for any $\varepsilon>0, J_{n}^{c, u}(s)$ will be near every point in the interval $[0,1]$ for some $s \in(t-\varepsilon, t+\varepsilon)$ and all suitably large $n$. In other words, the unit blur captures the increasing rate of fluctuations. In the present context, that is more important than knowing the distribution for any one fixed $t$, because the value at $t$ is not representative of the values for any time points near $t$, like white noise. Here the limit should be distributed something like uncountably many i.i.d. uniform random variables, which of course is not directly well defined. We should hope to obtain stronger results about integrals over finite intervals. Under additional regularity conditions, we should obtain the limit $\int_{a}^{b} \boldsymbol{J}_{n}^{c, u}(t) \mathrm{d} t \Rightarrow(b-a) / 2$ as $n \rightarrow \infty$.

\subsection{FCLTs with stronger scaling}

In this subsection we show that we can overcome the difficulties in Theorem 5.2 and obtain FCLTs for the counting processes, provided that we introduce a stronger scaling in the setting of Section 3.2. We will be brief and consider only the rate-1 processes with cyclic thinning, $S_{k}^{c, v}$ and $N^{c, v}(t)$. We introduce two new random elements in $D^{2}$, defined by

$$
\begin{aligned}
& \tilde{\boldsymbol{S}}_{n}^{c, v}(t) \equiv \frac{S_{n,\left\lfloor n^{2} t\right\rfloor}^{c, v}-\left\lfloor n^{2} t\right\rfloor}{\sqrt{n}}=\frac{S_{n\left\lfloor n^{2} t\right\rfloor}^{v}-n\left\lfloor n^{2} t\right\rfloor}{n^{3 / 2}} \approx \hat{S}_{n^{3}}^{v}(t), \\
& \tilde{\boldsymbol{N}}_{n}^{c, v}(t) \equiv \frac{N_{n}^{c, v}\left(n^{2} t\right)-n^{2} t}{\sqrt{n}}=\frac{N^{v}\left(n^{3} t\right)-n^{3} t-J_{n}^{v}\left(n^{3} t\right)}{n^{3 / 2}} \approx \hat{N}_{n^{3 / 2}}^{v}(t),
\end{aligned}
$$

for $t \geq 0$, using (5.3), where $\hat{\boldsymbol{S}}_{n}^{v}$ and $\hat{\boldsymbol{N}}_{n}^{v}$ are defined in (3.9) and (5.12), respectively. From the relations in (5.20), we obtain the following FCLT.

Theorem 5.4. (FCLT for counting processes with cyclic thinning and stronger scaling.) If $\hat{\boldsymbol{S}}_{n}^{v} \Rightarrow \hat{L}^{v}$ in $D$ for $\hat{\boldsymbol{S}}_{n}^{v}$ in (3.9), where $\mathrm{P}\left(\hat{L}^{v} \in C\right)=1$, as assumed in Theorem 5.1, then

$$
\left(\tilde{\boldsymbol{S}}_{n}^{c, v}, \tilde{\boldsymbol{N}}_{n}^{c, v}\right) \Rightarrow\left(\hat{L}^{v},-\hat{L}^{v}\right) \text { in } D^{2} \text { as } n \rightarrow \infty
$$

$\operatorname{for}\left(\tilde{\boldsymbol{S}}_{n}^{c, v}, \tilde{\boldsymbol{N}}_{n}^{c, v}\right)$ in $(5.20)$. 
Proof. For $\tilde{\boldsymbol{S}}_{n}^{c, v}$, apply (5.20) plus the reasoning in the proof of Theorem 4.2. For $\tilde{\boldsymbol{N}}_{n}^{c, v}$, first note that the $J_{n}^{v}$ term is asymptotically negligible, because $J_{n}^{v}(t)$ is bounded by $n$, but it is divided by $n^{3 / 2}$. Then apply (5.20) and then (5.11), which follows from (5.8), as noted in the proof of Theorem 5.1 .

\section{Queue length and workload processes}

As in Chapter 9 of [14], we can define other queueing processes. In the setting of Section 3.1, in model $n$ let $C_{n}(t)$ be the cumulative input of work, $X_{n}(t)$ be the net input of work, $I_{n}(t)$ be the cumulative idle time, $B_{n}(t)$ be the cumulative busy time, and $D_{n}(t)$ be the cumulative number of departures, all over the interval $[0, t]$ for $t \geq 0$. Let $Q_{n}(t)$ be the queue length (number in the system) and $R_{n}(t)$ be the remaining work in the system (the continuous-time workload), both at time $t$ for $t \geq 0$. These can be defined by

$$
\begin{aligned}
& C_{n}(t) \equiv S_{n, N_{n}^{u}(t)}^{v}, \quad X_{n}(t) \equiv C_{n}(t)-t, \quad I_{n}(t) \equiv-\inf _{0 \leq s \leq t}\left\{X_{n}(s)\right\}, \\
& B_{n}(t) \equiv t-I_{n}(t), \quad D_{n}(t) \equiv N_{n}^{v}\left(B_{n}(t)\right), \quad Q_{n}(t) \equiv N_{n}^{u}(t)-D_{n}(t), \\
& R_{n}(t) \equiv \psi\left(X_{n}\right)(t)=X_{n}(t)-\inf _{0 \leq s \leq t}\left\{X_{n}(s)\right\}=X_{n}(t)+I_{n}(t), \quad t \geq 0 .
\end{aligned}
$$

We add a superscript $c$ to indicate when these processes are associated with cyclic thinning, i.e. when the $n$th system is the $G_{n} / G_{n} / 1$ model.

\subsection{No time scaling}

In the setting of Section 5.1, we can establish limits for almost all the processes in (6.1). In particular, we establish limits for all but the queue length processes $Q_{n}^{c}$. For the queue length process, we are only able to establish a strong bound. In particular, we have the following result; we omit the proof for all but the queue length process, which follows easily from the basic definitions (4.1), (4.2), the assumption in (4.7), and the FWLLN following from (3.11).

Theorem 6.1. (HT limit in the $G_{n} / G_{n} / 1$ models without time scaling.) Assume that (5.8) is satisfied, again allowing either (4.7) or (3.12) to hold. Assume that $W_{n, 0}^{c} \Rightarrow W_{0}^{c}$. The following limit for the processes without time scaling holds: as $n \rightarrow \infty$,

$$
\begin{aligned}
& \left(S_{n}^{c, u}, S_{n}^{c, v}, S_{n}^{c}, W_{n}^{c}, N_{n}^{c, u}, N_{n}^{c, v}, C_{n}^{c}, X_{n}^{c}, I_{n}^{c}, B_{n}^{c}, R_{n}^{c}, D_{n}^{c}\right) \\
& \quad \Rightarrow\left(S^{c, u}, S^{c, v}, S^{c}, W^{c}, N^{c, u}, N^{c, v}, C^{c}, X^{c}, I^{c}, B^{c}, R^{c}, D^{c}\right)
\end{aligned}
$$

in $\left(\mathbb{R}^{\infty}\right)^{4} \times D^{8}$. Here

$$
\begin{gathered}
S_{k}^{c, u} \equiv k \equiv S_{k}^{c, v} \quad \text { and } \quad S_{k}^{c} \equiv W_{0}^{c} \equiv W_{k}^{c}, \quad k \geq 0, \quad N^{c, u}(t) \equiv 1+\lfloor t\rfloor, \\
N^{c, v}(t) \equiv\lfloor t\rfloor, \quad C^{c}(t) \equiv W_{0}^{c}+1+\lfloor t\rfloor, \quad X^{c}(t) \equiv W_{0}^{c}+1+\lfloor t\rfloor-t, \\
I^{c}(t) \equiv 0, \quad B^{c}(t) \equiv t, \quad R^{c}(t) \equiv X^{c}(t), \quad D^{c}(t) \equiv\lfloor t\rfloor .
\end{gathered}
$$

In addition, if $W_{0}^{c} \equiv 0$ then, for all noninteger $t, Q_{n}^{c}(t) \Rightarrow 1$ as $n \rightarrow \infty$ and

$$
\mathrm{P}\left(Q_{n}^{c}(s) \in\{0,1,2\} \text { for all } s \in[0, t]\right) \rightarrow 1 \text { as } n \rightarrow \infty \text { for all } t>0 .
$$

Proof. We only have difficulty with $Q_{n}^{c}$. For it, we avoid specifying the number of customers in the system initially by assuming that $W_{n, 0}^{c} \Rightarrow 0$. Still $Q_{n}^{c}$ remains difficult because it involves subtraction of integer-valued functions with common discontinuity points. We have 
$Q_{n}^{c}(t) \equiv N_{n}^{c, u}(t)-D_{n}^{c}(t)$, where $D_{n}^{c}(t)=N_{n}^{c, v}\left(B_{n}^{c}(t)\right) \Rightarrow\lfloor e\rfloor$. The limit for noninteger $t$ is thus $(1+\lfloor e\rfloor)(t)-(\lfloor e\rfloor)(t)=1$. But there can be problems at the integer points where both limits have discontinuities. But at each discontinuity point the error can be at most 1 . Hence, we have (6.2).

If, instead of the initial conditions given in Theorem 6.1, we had the arrival at time 0 find $k-1$ others in the system at time 0 , with one of those just beginning service, so that $Q^{c}(0)=k$, including the new arrival, we would have the alternative limits $Q^{c}(t)=k$ and $R^{c}(t)=k+\lfloor t\rfloor-t$ for all noninteger time points $t>0$, so that $k-1 \leq R^{c}(t) \leq k$ and $k-1 \leq Q(t) \leq k+1$ for all $t \geq 0$. The practical implication is that the $G_{n} / G_{n} / 1$ model for large $n$ behaves like the $\mathrm{D} / \mathrm{D} / 1$ model with $\rho=1$ over short time intervals. (Note that, with deterministic unit service times, $Q(t)=\lceil W(t)\rceil$, where $\lceil t\rceil$ is the ceiling function, giving the least integer greater than $t$.) Consequently, the queue length and workload processes in the $\mathrm{G}_{n} / \mathrm{G}_{n} / 1$ model for large $n$ will change negligibly over short time intervals.

\subsection{Time scaling by $n$}

We now show the implications of the results in Section 5.2 for the other queueing processes introduced in (6.1). Define the following random elements of $D$ :

$$
\begin{aligned}
\boldsymbol{X}_{n}^{c}(t) & \equiv \boldsymbol{C}_{n}^{c}(t) \equiv C^{c}(n t)-n t=X^{c}(n t), \\
\boldsymbol{R}_{n}^{c}(t) & \equiv R_{n}^{c}(n t)=\phi\left(\boldsymbol{X}_{n}^{c}\right)(t), \\
\boldsymbol{I}_{n}^{c}(t) & \equiv I_{n}^{c}(n t)=n t-B_{n}^{c}(n t) \equiv-\boldsymbol{B}_{n}^{c}(t), \\
\boldsymbol{D}_{n}^{c}(t) & \equiv D_{n}^{c}(n t)-n t=N_{n}^{c, v}\left(B_{n}^{c}(n t)\right)-n t, \\
\boldsymbol{Q}_{n}^{c}(t) & \equiv Q_{n}^{c}(n t)=N_{n}^{c, u}(n t)-D_{n}^{c}(n t)=N_{n}^{c, u}(t)-D_{n}^{c}(t) .
\end{aligned}
$$

Theorem 6.2. (HT limit for other processes in the $\mathrm{G}_{n} / \mathrm{G}_{n} / 1$ model.) Under the assumptions of Theorem 5.1,

$$
\left(\boldsymbol{W}_{n}^{c}, \boldsymbol{X}_{n}^{c}, \boldsymbol{R}_{n}^{c}, \boldsymbol{B}_{n}^{c}, \boldsymbol{I}_{n}^{c}, \boldsymbol{D}_{n}^{c}, \boldsymbol{Q}_{n}^{c}\right) \Rightarrow\left(W^{c}, X^{c}, R^{c}, B^{c}, I^{c}, D^{c}, Q^{c}\right) \quad \text { in } \boldsymbol{C}^{7},
$$

where

$$
\begin{gathered}
W^{c} \equiv \phi(L), \quad X^{c} \equiv L+\Upsilon^{u}, \quad R^{c} \equiv \phi(L)+\Upsilon^{u}, \quad B^{c}=-I^{c} \equiv L-\phi(L), \\
D^{c} \equiv-\hat{L}^{v}-\Upsilon^{v}+L-\phi(L), \quad \text { and } \quad Q^{c} \equiv \phi(L)+\Upsilon^{u}+\Upsilon^{v},
\end{gathered}
$$

$L \equiv \hat{L}^{v}-\hat{L}^{u}-\beta e$ for $\left(\hat{L}^{u}, \hat{L}^{v}\right)$ in (3.11), $\phi$ is the reflection map in (3.6), and $\Upsilon^{u}$ and $\Upsilon^{v}$ are the unit blurs associated with $N_{n}^{c, u}$ and $N_{n}^{c, v}$, respectively.

Proof. We first obtain $\boldsymbol{W}_{n}^{c} \Rightarrow W^{c} \equiv \phi(L)$ in $D$ and, thus, in $\boldsymbol{C}$ from Theorem 4.2. Jointly with that, we can obtain the following limits. Note that $\boldsymbol{X}_{n}^{c}=\boldsymbol{S}_{n}^{c, v} \circ \overline{\boldsymbol{N}}_{n}^{c, u}+\boldsymbol{N}_{n}^{c, u}$, where $\left(\boldsymbol{S}_{n}^{c, v}, \overline{\boldsymbol{N}}_{n}^{c, u}, \boldsymbol{N}_{n}^{c, u}\right) \Rightarrow\left(\hat{L}^{v}, e,-\hat{L}^{u}-\beta e+\Upsilon^{u}\right)$. Hence, $\boldsymbol{X}_{n}^{c} \Rightarrow L+\Upsilon^{u}$ as $n \rightarrow \infty$. Then $\boldsymbol{R}_{n}^{c}(t) \Rightarrow \phi\left(L+\Upsilon^{u}\right)=\phi(L)+\Upsilon^{u}$ and $\boldsymbol{I}_{n}^{c}=\boldsymbol{X}_{n}^{c}-\boldsymbol{R}_{n}^{c} \Rightarrow L-\phi(L)$. Next, for $\boldsymbol{D}_{n}^{c}$, first note that $\overline{\boldsymbol{B}}_{n}^{c} \Rightarrow e$, where $\overline{\boldsymbol{B}}_{n}^{c}(t) \equiv B_{n}^{c}(n t) / n$, because $\boldsymbol{B}_{n}^{c}$ converges to a nondegenerate limit. Since $\boldsymbol{D}_{n}^{c}=\boldsymbol{N}_{n}^{c, v} \circ \overline{\boldsymbol{B}}_{n}^{c}+\boldsymbol{B}_{n}^{c}, \boldsymbol{D}_{n}^{c} \Rightarrow-\hat{L}^{v}-\Upsilon^{v}+L-\phi(L)$ and $\boldsymbol{Q}_{n}^{c}=\boldsymbol{N}_{n}^{c, u}-\boldsymbol{D}_{n}^{c} \Rightarrow-\hat{L}^{u}-\beta e+$ $\Upsilon^{u}+\hat{L}^{v}+\Upsilon^{v}-L+\phi(L)=\phi(L)+\Upsilon^{u}+\Upsilon^{v}$.

Note that as a special case of the limit in (6.4) above, we have the joint limit

$$
\left(\boldsymbol{W}_{n}^{c}, \boldsymbol{R}_{n}^{c}, \boldsymbol{Q}_{n}^{c}\right) \Rightarrow\left(W^{c}, R^{c}, Q^{c}\right) \equiv\left(\phi(L), \phi(L)+\Upsilon^{u}, \phi(L)+\Upsilon^{u}+\Upsilon^{v}\right) \quad \text { in } \boldsymbol{C}^{3},
$$


from which we see that the limits are ordered, i.e.

$$
W^{c}(t) \leq R^{c}(t) \leq W^{c}(t)+1 \quad \text { and } \quad R^{c}(t) \leq Q^{c}(t) \leq R^{c}(t)+1 \quad \text { for all } t \geq 0,
$$

with strict inequality holding for most $t$. We will develop explicit approximations for the associated steady-state quantities $\left(W_{n, \infty}^{c}, R_{n, \infty}^{c}, Q_{n, \infty}^{c}\right)$ in [11], which refine (6.5) but are consistent with it.

\subsection{Time scaling by $n^{2}$}

We now show the implications of Theorem 5.4 for the other queueing processes introduced in (6.1); we omit the elementary proof. Define the following new random elements of $D$ by scaling time by $n$ and dividing by $\sqrt{n}$ in the previous random elements in (6.3):

$$
\begin{aligned}
\tilde{\boldsymbol{X}}_{n}^{c}(t) & \equiv \tilde{\boldsymbol{C}}_{n}^{c}(t) \equiv \frac{\boldsymbol{X}_{n}^{c}(n t)}{\sqrt{n}}=\frac{X^{c}\left(n^{2} t\right)}{\sqrt{n}} \\
\tilde{\boldsymbol{R}}_{n}^{c}(t) & \equiv \frac{\boldsymbol{R}_{n}^{c}(n t)}{\sqrt{n}}=\phi\left(\tilde{\boldsymbol{X}}_{n}^{c}\right)(t) \\
\tilde{\boldsymbol{I}}_{n}^{c}(t) & \equiv \frac{\boldsymbol{I}_{n}^{c}(n t)}{\sqrt{n}} \\
\tilde{\boldsymbol{D}}_{n}^{c}(t) & \equiv \frac{\boldsymbol{D}_{n}^{c}(n t)}{\sqrt{n}} \\
\tilde{\boldsymbol{Q}}_{n}^{c}(t) & \equiv \frac{\boldsymbol{Q}_{n}^{c}(n t)}{\sqrt{n}}=\tilde{\boldsymbol{N}}_{n}^{c, u}(t)-\tilde{\boldsymbol{D}}_{n}^{c}(t)
\end{aligned}
$$

Overall, time is now scaled by $n^{2}$, e.g. $\tilde{Q}_{n}^{c}(t)=Q_{n}^{c}\left(n^{2} t\right) / \sqrt{n}, t \geq 0$.

Theorem 6.3. (HT limit for other processes in the $\mathrm{G}_{n} / \mathrm{G}_{n} / 1$ model with stronger scaling.) Under the assumptions of Theorem 5.1,

$$
\left(\tilde{\boldsymbol{W}}_{n}^{c}, \tilde{\boldsymbol{X}}_{n}^{c}, \tilde{\boldsymbol{R}}_{n}^{c}, \tilde{\boldsymbol{B}}_{n}^{c}, \tilde{\boldsymbol{I}}_{n}^{c}, \tilde{\boldsymbol{D}}_{n}^{c}, \tilde{\boldsymbol{Q}}_{n}^{c}\right) \Rightarrow\left(\tilde{W}^{c}, \tilde{X}^{c}, \tilde{R}^{c}, \tilde{B}^{c}, \tilde{I}^{c}, \tilde{D}^{c}, \tilde{Q}^{c}\right) \text { in } D^{7}
$$

where

$$
\begin{gathered}
\tilde{W}^{c} \equiv \phi(L), \quad \tilde{X}^{c} \equiv L, \quad \tilde{R}^{c} \equiv \phi(L), \quad \tilde{B}^{c}=-\tilde{I}^{c} \equiv L-\phi(L), \\
\tilde{D}^{c} \equiv-\hat{L}^{v}+L-\phi(L), \quad \text { and } \quad \tilde{Q}^{c} \equiv \phi(L),
\end{gathered}
$$

$L \equiv \hat{L}^{v}-\hat{L}^{u}-\beta e$ for $\left(\hat{L}^{u}, \hat{L}^{v}\right)$ in (3.11), and $\phi$ is the reflection map in (3.6).

\section{The motivating $G_{n} / D / \infty$ infinite-server queue}

We now apply the FCLT just established in Section 5.3 to obtain an HT FCLT for the queue length (number of busy servers) in a $\mathrm{G}_{n} / \mathrm{D} / \infty$ model, addressing the motivating inventory problem from [12] mentioned in Section 2. We start with unit service times and a rate- 1 arrival process; let the counting process be $\left\{N^{u}(t): t \geq 0\right\}$. To achieve an associated rate- $\lambda$ process, we scale time by $\lambda$, i.e. by using the process $\left\{N^{u}(\lambda t)\right\}$. Starting with a base $\mathrm{G} / \mathrm{D} / \infty$ queue with a rate- 1 arrival process, we want to consider the associated $G_{n} / D / \infty$ queue with an arrival rate of $n^{2}$. We denote this arrival process as $\left\{N_{n}^{c, u}(t): t \geq 0\right\}$. (Motivated by [12], the arrival rate should grow as the square of the cycle order $n$.) 
Let the queue length at time $t$ with arrival process $N_{n}^{c, u}$ and arrival rate $n^{2}$ be $Q_{n}^{\infty, c}(t)$. As in [5],

$$
Q_{n}^{\infty, c}(t)=N_{n}^{c, u}(t)-N_{n}^{c, u}(t-1) \quad \text { for all } t \geq 1 .
$$

Consequently, if the arrival process is time stationary then $Q_{n}^{\infty, c}(t)$ has a fixed (stationary) distribution for all $t \geq 1$, regardless of the initial conditions. (It reaches steady state at time 1.) In general, we can obtain an HT FCLT for the scaled queue length process

$$
Q_{n}^{\infty, c}(t) \equiv \frac{Q_{n}^{\infty, c}(t)-n^{2}}{\sqrt{n}}, \quad t \geq 0 .
$$

Theorem 7.1. For the sequence of $G_{n} / D / \infty$ queues with arrival rate $n^{2}$ in model $n$ (with cyclic thinning of order $n$ ) just defined, if the base stationary arrival counting process $N^{u}$ satisfies an FCLT, i.e. if $\hat{N}_{n}^{u} \Rightarrow \sigma_{u} B$ in $D$, where $B$ is $B M$ and $N_{n}^{u}(t) \equiv\left(N^{u}(n t)-n t\right) / \sqrt{n}$, then

$$
\boldsymbol{Q}_{n}^{\infty, c} \Rightarrow \boldsymbol{Q}^{\infty, c} \quad \text { in } D([1, \infty), \mathbb{R}),
$$

where $\boldsymbol{Q}_{n}^{\infty, c}$ is defined in (7.2) above and $\boldsymbol{Q}^{\infty, c}(t) \equiv \sigma_{u}(B(t)-B(t-1), t \geq 1$, so that

$$
\frac{Q_{n}^{\infty, c}(t)-n^{2}}{\sqrt{n}} \Rightarrow N\left(0, \sigma_{u}^{2}\right) \quad \text { in } \mathbb{R} \text { as } n \rightarrow \infty \text { for all } t \geq 1 \text {. }
$$

Proof. Essentially, we are applying Theorem 5.4. Directly, by (7.1) and the fact that $N_{n}^{u, c}$ has rate $n^{2}$,

$$
\begin{aligned}
Q_{n}^{\infty, c}(t) & \stackrel{\mathrm{D}}{=} N_{n}^{c, u}(t)-N_{n}^{c, u}((t-1)) \\
& =\frac{N^{u}\left(n^{3} t\right)-J_{n}^{c, u}\left(n^{3} t\right)-N^{u}\left(n^{3}(t-1)\right)+J_{n}^{c, u}\left(n^{3}(t-1)\right)}{n} .
\end{aligned}
$$

Hence, when we divide by $\sqrt{n}$ in (7.5), as required for (7.2), the two $J_{n}^{c, u}$ terms on the righthand side of (7.5) become asymptotically negligible. Ignoring them (using Theorems 11.4.5 and 11.4.7 of [14]), we have $\boldsymbol{Q}_{n}^{\infty, c}(t) \stackrel{\mathrm{D}}{=} \boldsymbol{N}_{n^{3}}^{u}(t)-\boldsymbol{N}_{n^{3}}^{u}(t-1)$. Hence, the limit in (7.3) follows from the assumed FCLT for $N_{n}^{u}$.

Because of the cyclic thinning, in model $n$ the translation term in (7.2) is $n^{2}$, but the spatial scaling is only by $\sqrt{n}$. Nevertheless, this is consistent with [5] (even though it does not follow directly from [5]); e.g. for the $\mathrm{G}_{k} / \mathrm{D} / \infty$ model, with arrival rate $n^{2}$ but fixed cyclic thinning order $k$, from [5] we get the limit

$$
\frac{Q_{n}^{\infty, c, k}(t)-n^{2}}{n} \Rightarrow N\left(0, \frac{\sigma_{u}^{2}}{k}\right) \stackrel{\mathrm{D}}{=} \frac{1}{\sqrt{k}} N\left(0, \sigma_{u}^{2}\right) \quad \text { in } \mathbb{R} \text { as } n \rightarrow \infty
$$

for each fixed $k$. If we multiply through by $\sqrt{k}$ in (7.6) and then (formally) let $n \rightarrow \infty$ with $k=n$, we obtain (7.4).

\section{Acknowledgements}

This research was supported by NSF grant CMMI 0948190. We thank doctoral students Yunan Liu for conducting supporting simulation experiments and Guodong Pang for helpful comments. 


\section{References}

[1] Aвate, J., Choudhury, G. L. and Whitt, W. (1993). Calculation of the GI/G/1 steady-state waiting-time distribution and its cumulants from Pollaczek's formula. Internat. J. Electronics Commun. 47, 311-321.

[2] Asmussen, S. (2003). Applied Probability and Queues, 2nd edn. Springer, New York.

[3] Billingsley, P. (1999). Convergence of Probability Measures, 2nd edn. John Wiley, New York.

[4] Borovkov, A. A. (1984). Asymptotic Methods in Queuing Theory. John Wiley, Chichester.

[5] Glynn, P. W. AND Whitt, W. (1991). A new view of the heavy-traffic limit theorem for the infinite-server queue. Adv. Appl. Prob. 23, 188-209.

[6] Halfin, S. And Whitt, W. (1981). Heavy-traffic limits for queues with many exponential servers. Operat. Res. 29, 567-588.

[7] Jelenkovic, P., Mandelbaum, A. And MomČIlović, P. (2004). Heavy traffic limits for queues with many deterministic servers. Queueing Systems 47, 53-69.

[8] Kingman, J. F. C. (1961). The single-server queue in heavy traffic. Proc. Camb. Phil. Soc. 57, 902-904.

[9] LiU, Y. And Whitt, W. (2011). Nearly periodic behavior in the overloaded G/D/S+GI queue. Stoch. Systems 1, $71 \mathrm{pp}$.

[10] Pang, G., Talreja, R. and Whitt, W. (2007). Martingale proofs of many-server heavy-traffic limits for Markovian queues. Prob. Surveys 4, 193-267.

[11] Sigman, K. And Whitt, W. (2011). Heavy-traffic limits for nearly deterministic queues: stationary distributions. To appear in Queueing Systems.

[12] Song, J.-S. AND Zipkin, P. H. (1996). The joint effect of leadtime variance and lot size in a parallel processing environment. Manag. Sci. 42, 1352-1363.

[13] Tisms, H. C. (1994). Stochastic Models. John Wiley, Chichester.

[14] Whitт, W. (2002). Stochastic-Process Limits. Springer, New York. 